Draft Version September 5, 2018

Preprint typeset using $\mathrm{L}_{\mathrm{A}} \mathrm{EX}$ style emulateapj v. 5/2/11

\title{
CLASSIFICATION OF X-RAY SOURCES IN THE XMM-NEWTON SERENDIPITOUS SOURCE CATALOG: OBJECTS OF SPECIAL INTEREST
}

\author{
Dacheng Lin $^{1,2,3}$, NAtalie A. WebB ${ }^{1,2}$, Didier Barret ${ }^{1,2}$ \\ Draft version September 5, 2018
}

\begin{abstract}
We analyze 18 sources that were found to show interesting properties of periodicity, very soft spectra, and/or large long-term variability in X-rays in our project of classification of sources from the 2XMMi-DR3 catalog but were poorly studied in the literature, in order to investigate their nature. Two hard sources show X-ray periodicities of $\sim 1.62 \mathrm{hr}(2 \mathrm{XMM}$ J165334.4-414423) and $\sim 2.1$ hr (2XMM J133135.2-315541) and are probably magnetic cataclysmic variables. One source 2XMM $\mathrm{J} 123103.2+110648$ is an active galactic nucleus (AGN) candidate showing very soft X-ray spectra $(k T \sim 0.1 \mathrm{keV})$ and exhibiting an intermittent $\sim 3.8 \mathrm{hr}$ quasi-periodic oscillation. There are six other very soft sources (with $k T<0.2 \mathrm{keV}$ ), which might be in other galaxies with luminosities between $\sim 10^{38}-10^{42} \mathrm{erg} \mathrm{s}^{-1}$. They probably represent a diverse group that might include objects such as ultrasoft AGNs and cool thermal disk emission from accreting intermediate-mass black holes. Six highly variable sources with harder spectra are probably in nearby galaxies with luminosities above $10^{37} \mathrm{erg} \mathrm{s}^{-1}$ and thus are great candidates for extragalactic X-ray binaries. One of them (2XMMi J004211.2+410429, in M 31) is probably a new-born persistent source, having been X-ray bright and hard in $0.3-10 \mathrm{keV}$ for at least four years since it was discovered to enter an outburst in 2007 . Three highly variable hard sources appear at low galactic latitudes and have maximum luminosities below $\sim 10^{34} \mathrm{erg} \mathrm{s}^{-1}$ if they are in our Galaxy, thus great candidates for cataclysmic variables or very faint $\mathrm{X}$-ray transients harboring a black hole or neutron star. Our interpretations of these sources can be tested with future long-term X-ray monitoring and multi-wavelength observations.
\end{abstract}

Subject headings: accretion, accretion disks — stars: neutron - X-rays: binaries - X-rays: bursts - X-ray: stars

\section{INTRODUCTION}

In Lin et al. (2012b, LWB12 hereafter), we carried out source type classifications of 4330 sources from the 2XMMi-DR3 catalog, which is the largest X-ray source catalog ever produced, containing 353,191 Xray source detections for 262,902 unique $\mathrm{X}$-ray sources from 4953 pointed observations made by XMM-Newton (Watson et al. 2009). For about one third of these 4330 sources we obtained reliable source types from the literature. They mostly correspond to various types of stars, active galactic nuclei (AGNs) and compact object (CO) systems containing white dwarfs (WDs), neutron stars (NSs), and stellar-mass black holes (BHs). These sources show different source properties in terms of the $\mathrm{X}$-ray spectral shape, X-ray variability, and the multiwavelength cross-correlation, based on which we came up with a method to classify the rest of the sources into the above three main categories, i.e., stars, AGNs, and compact object systems. In brief, we first identified star candidates as those with low X-ray-to-IR flux ratios and/or frequent X-ray flares and then identified compact object system candidates from the remaining sources as those with properties hardly seen in AGNs, such as soft X-ray spectra and large long-term variability. The compact object system candidates identified

\footnotetext{
${ }^{1}$ CNRS, IRAP, 9 avenue du Colonel Roche, BP 44346, F31028 Toulouse Cedex 4, France

${ }^{2}$ Université de Toulouse, UPS-OMP, IRAP, Toulouse, France

${ }^{3}$ Department of Physics and Astronomy, University of Alabama, Box 870324, Tuscaloosa, AL 35487, USA, email: dlin@ua.edu
}

in this way could inevitably include exotic objects with high science value, such as very soft AGNs like GSN 069 (Miniutti et al. 2013) and tidal disruption events (TDEs), in which stars approaching a supermassive BH (SMBH) is tidally disrupted and subsequently accreted (Lidskii \& Ozernoi 1979; Rees 1988).

About 200 strong compact object system candidates were found. Some of them were poorly studied in the literature. Because these objects are important targets for studies of dense matter and accretion physics in extreme environment and they are also a treasure for discovery of objects with high science value, we have planned a series of companion papers on these poorly studied compact object system candidates in order to shed new light on their nature. While the other companion papers focus on single sources (e.g., Lin et al. 2011, 2013b), here we present a relatively large sample (18 in total), which were selected because they exhibited interesting properties of periodicity, very soft spectra, and/or large longterm variability. Some of their general properties from LWB12 are given in Table 1 including the number of source detections in the 2XMMi-DR3 catalog, the number of the XMM-Newton observations, the $0.2-4.5 \mathrm{keV}$ flux variation $\left(V_{\text {var14 }}\right)$, etc. Throughout the paper, we generally use the unique source index from the $2 \mathrm{XMMi}-$ DR3 catalog (column SRCID) to refer to the sources (Table 11).

In this paper, we concentrate primarily on $X M M$ Newton X-ray observations. We only present those that best demonstrate the source properties based on considerations of data quality, sampling of spectral states, etc. 
Lin et al.
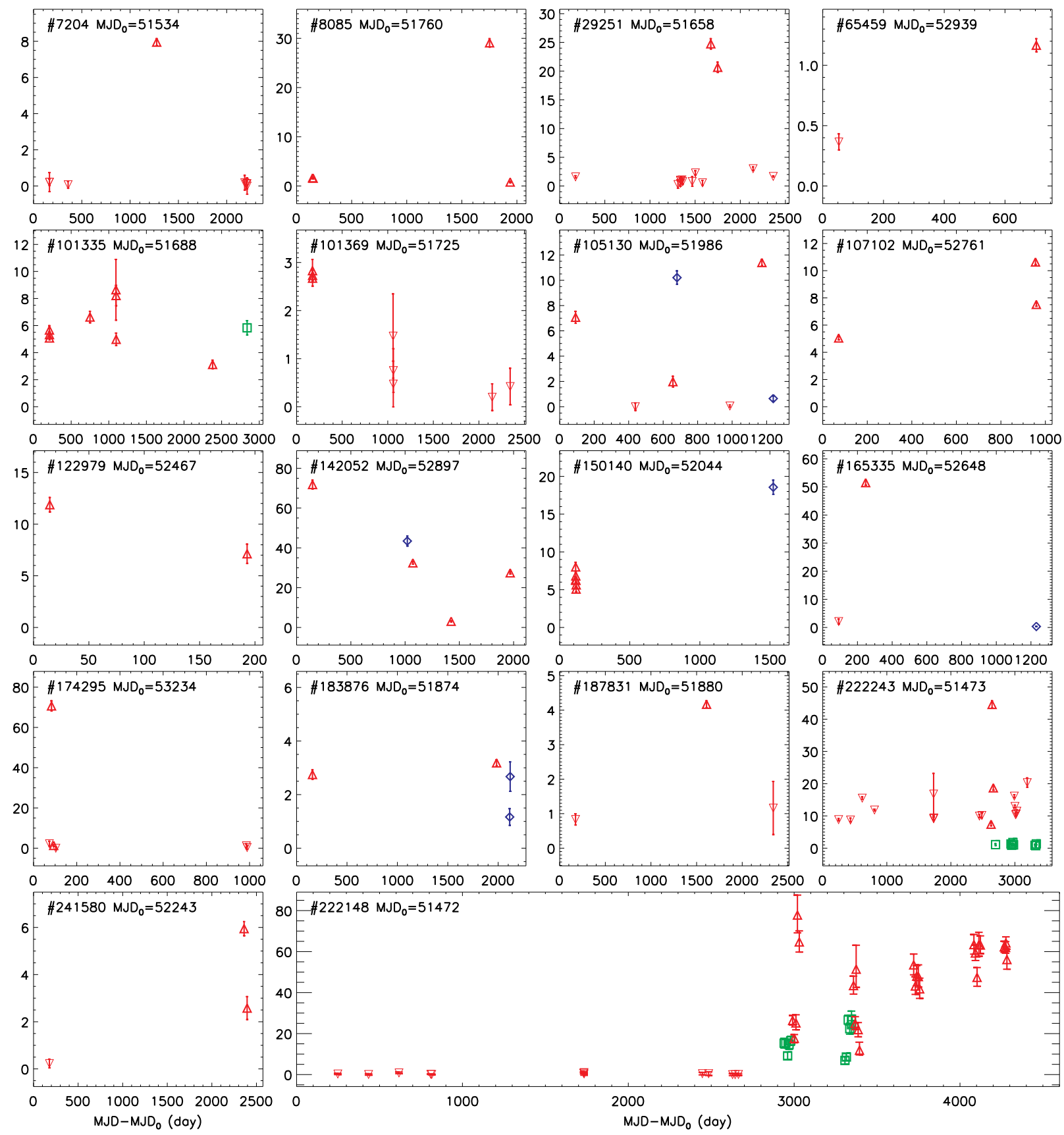

Fig. 1. - The long-term flux curve (in units of $10^{-14} \mathrm{erg} \mathrm{s}^{-1} \mathrm{~cm}^{-2}$ ). The $0.2-4.5 \mathrm{keV}$ fluxes are plotted for XMM-Newton observations; those when the source was detected are from the 2XMMi-DR3 catalog (red triangles; for source \#222148, the 0.3-10 keV absorbed fluxes from our spectral fits are used (Table 31), while those when the source was not detected were estimated from http://www.ledas.ac.uk/flix/flix.html (upside down triangles). For Chandra observations only detections from the CSC are plotted, with the 0.5-7 keV Huxes plotted tor ACIS observations (blue diamonds) and 0.1-10 keV fluxes for High Resolution Camera (HRC) observations (green squares).

Some sources are also observed by other X-ray observatories, especially the Chandra X-ray Observatory (CXO). We mostly refer to the Chandra Source Catalog (CSC, release 1.1, Evans et al. 2010) for their behavior as observed with Chandra, except for one observation, which was analyzed to show the period of source \#150140. In Section 2, we describe the analysis of the selected $X M M$ Newton and Chandra observations of the sources. In Section [3. we present the results and discuss the possible nature of each source. The conclusions of our study are given in Section 4. Throughout the paper, we assume a flat universe with the Hubble constant $H_{0}=73 \mathrm{~km} \mathrm{~s}^{-1}$
$\mathrm{Mpc}^{-1}$ and the matter density $\Omega_{\mathrm{M}}=0.27$.

\section{DATA ANALYSIS}

The details of the XMM-Newton observations that we analyzed are given in Table 2. We used SAS 11.0.0 and the calibration files of 2011 June for reprocessing the $\mathrm{X}$-ray event files and follow-up analysis. The data in strong background flare intervals are excluded following the SAS thread for filtering against high background. We extracted the source spectrum for all available cameras from a circular region centered on the source, with the radius $r_{\mathrm{src}}$ for each observation given in Table 2. The 


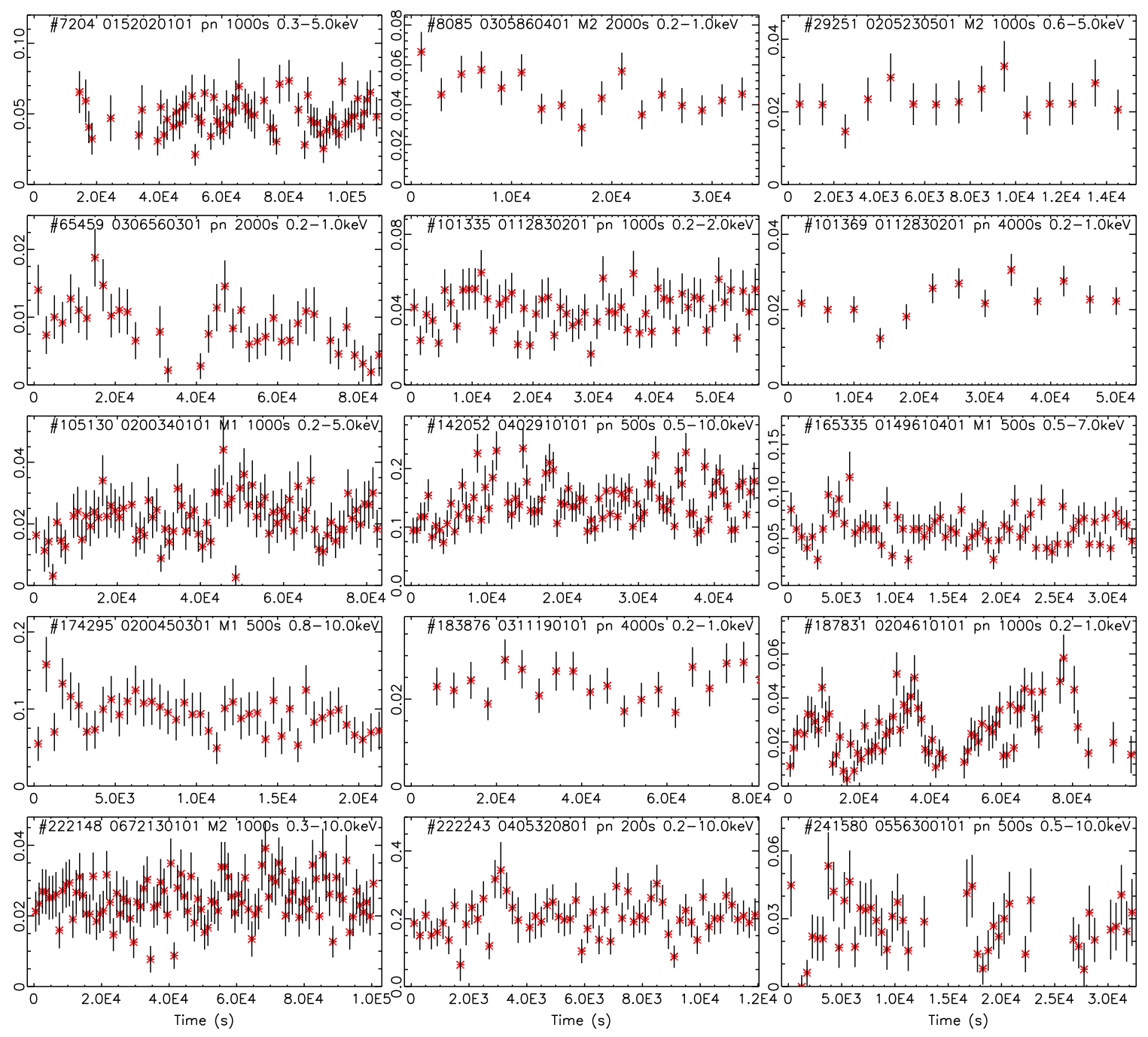

FIG. 2.- The light curves (in units of $\mathrm{cts} \mathrm{s}^{-1}$ ) of bright detections. In each panel, we note the SRCID number, the observation ID, the EPIC camera, the bin time, and the energy band used to produce each light curve.

TABLE 1

General Source Properties from LWB12

\begin{tabular}{|c|c|c|c|c|c|c|c|c|c|c|c|c|c|c|c|c|c|c|c|}
\hline $\begin{array}{r}\text { RCID } \\
(1) \\
\end{array}$ & $\begin{array}{l}\text { MMi-DR3 } \\
\text { (2) }\end{array}$ & $\begin{array}{l}\text { ePos } \\
(3)\end{array}$ & $\begin{array}{l}\text { GLA? } \\
(4)\end{array}$ & $\begin{array}{c}\text { NObs } \\
(5)\end{array}$ & $\begin{array}{c}\text { DObs } \\
(6)\end{array}$ & $\begin{array}{l}\text { NDet } \\
(7)\end{array}$ & $\begin{array}{c}\mathrm{DD} \text { Det } \\
(8)\end{array}$ & $\begin{array}{l}\text { Type } \\
(9)\end{array}$ & $\begin{array}{l}\text { reChar } \\
(10)\end{array}$ & $\begin{array}{l}\text { Dxo } \\
(11)\end{array}$ & $\begin{array}{c}R 2 \\
(12) \\
\end{array}$ & $\begin{array}{l}\text { Rxo } \\
(13)\end{array}$ & $\begin{array}{l}\text { xir } \\
14)\end{array}$ & $\begin{array}{c}K_{\mathrm{S}} \\
(15)\end{array}$ & $\begin{array}{l}\text { Rxir } \\
(16)\end{array}$ & $\begin{array}{c}F \max \\
(17)\end{array}$ & (18) & $\begin{array}{l}\text { C3Nam } \\
\text { (19) }\end{array}$ & $\begin{array}{l}\mathrm{C} 3 \mathrm{Se} \\
(20)\end{array}$ \\
\hline $72042 \mathrm{XN}$ & I J00470 & 4 & -88.0 & 9 & 47.70 & 1 & 0 & $\mathrm{CO}$ & $\mathrm{s}$ & & $\ldots$ & 1 & . & . & 1 & 0.5 & 8 & $\mathrm{NGC2}$ & 0.74 \\
\hline & & & & & & & & & & & & & & & & & & & \\
\hline 292512 & $\mathrm{~J}$ & & -4 & 14 & & 2 & 9 & & , & & $\cdots$ & & $\cdots$ & & & & & NGE & \\
\hline 654592 & I $\mathrm{JO}$ & & -2 & & 7 & 1 & & & & $\ldots$ & $\cdots$ & -0.12 & $\cdots$ & $\cdots$ & -0.82 & 1.3 & & & \\
\hline 5 & $\mathrm{~J}$ & & & & & 8 & 169.02 & & & & & & $\cdots$ & 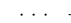 & & & & & \\
\hline 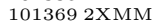 & & & & 8 & & 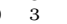 & & & & .5 & 19.33 & & $\ldots$ & $\ldots$ & & & & & \\
\hline 02 & & & & & & & 108 & & $\mathrm{~S}, \mathrm{~V}, \mathrm{E}$ & & & & $\cdots$ & $\cdots$ & & 0.4 & & $\mathrm{GC}$ & \\
\hline 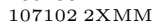 & & & & & & & & & & 3.6 & 19.72 & & & & & & & & \\
\hline 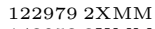 & & & & 2 & & & & & & & & & & & & & & & \\
\hline 22 & & & -6 & 4 & & 4 & & & & & 16.82 & & 1.3 & 13.76 & & & & & \\
\hline 2 & & & & & & & & & & & & & & & & & & & \\
\hline 52 & & & -2 & & & & & & & & 20 & & & 12 & & & & & \\
\hline $52 x$ & & 0 & & 6 & & 2 & & & & 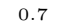 & 19.70 & & 0.2 & 14.01 & & & & & \\
\hline $625-3 x-10$ & & & -5 & & & & & $3 \mathrm{UI}$ & & & & & & & & & & & 0.82 \\
\hline & & & & & & & & $\mathrm{UI}$ & & & $\cdots$ & & & . & & & & & \\
\hline $2 \mathrm{x}$ & & 0.3 & -2 & 1 & & 5 & & & $\mathrm{~V}$ & $\cdots$ & $\cdots$ & & $\ldots$ & $\cdots$ & & & & & \\
\hline $2 x$ & & 0.3 & -21 & & & & & ( & & & $\cdots$ & & 0.8 & 14.43 & & & & & \\
\hline $02 \mathrm{XMI}$ & Ii J123047.0+413651 & 0.4 & 74.9 & 3 & 217.86 & 2 & 33.89 & $\mathrm{CO}$ & $V, E$ & & 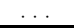 & 0.80 & & & 0.1 & & & NGC4490 & 0.87 \\
\hline \multicolumn{20}{|c|}{ 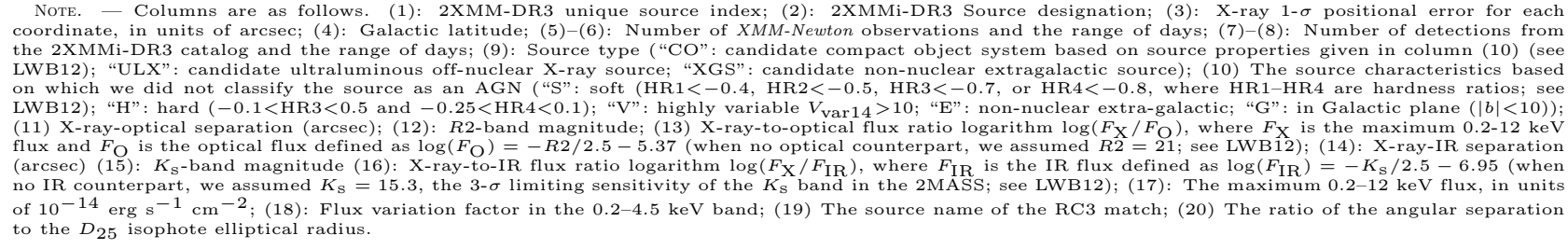 } \\
\hline
\end{tabular}




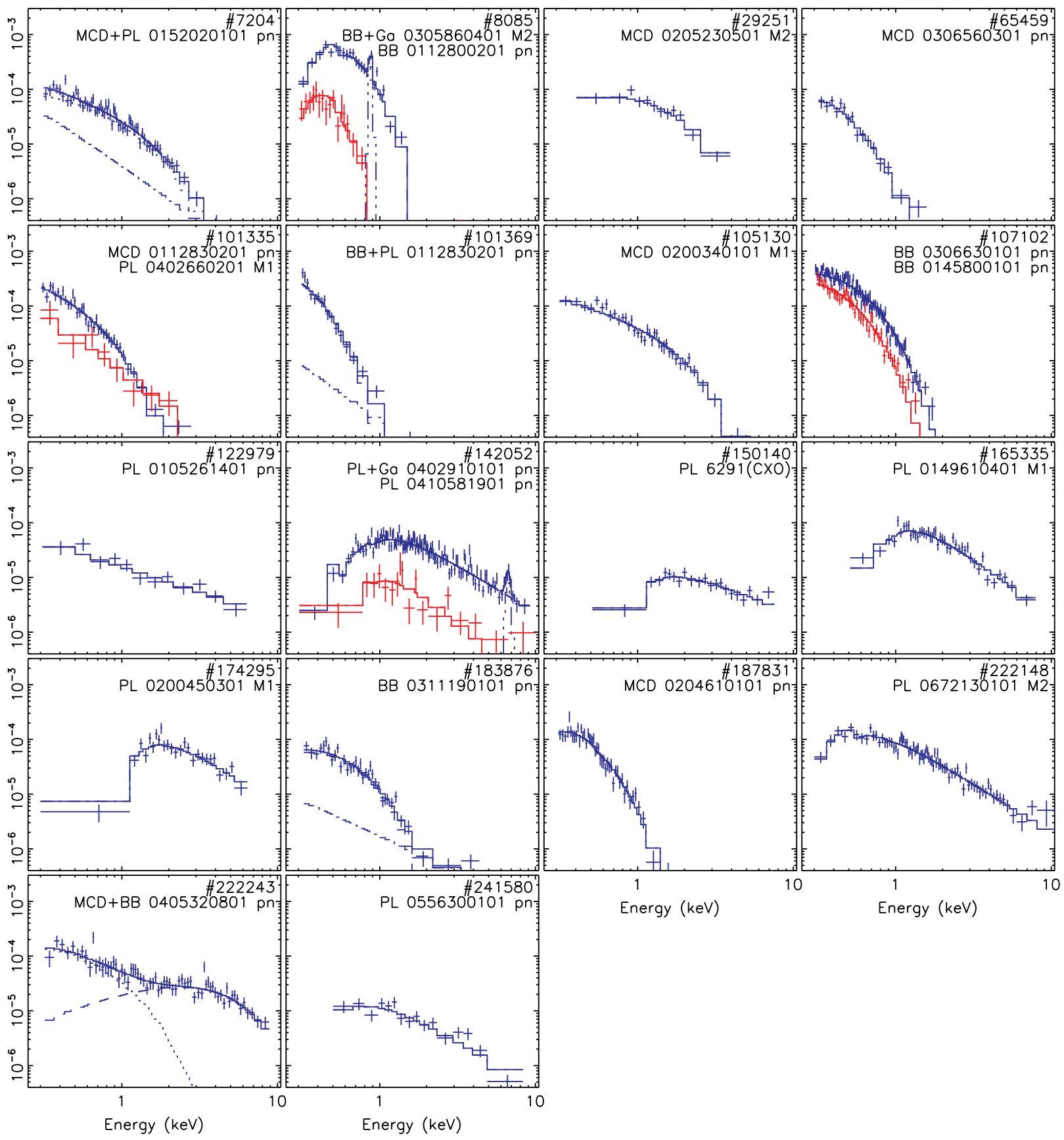

FIG. 3. - The unfolded spectra (in units of photons $\mathrm{cm}^{-2} \mathrm{~s}^{-1} \mathrm{keV}^{-1}$ ) of some spectral fits. In each panel, we note the SRCID number, the spectral model, the observation ID, and the EPIC camera used in the plot. For panels with multiple spectra, the observation IDs are given in the order of the spectra from the top to the bottom. The dotted, dashed, dot-dashed,dot-dot-dot-dashed, and solid lines correspond to the MCD, BB, PL, Gaussian line, and the total models. 

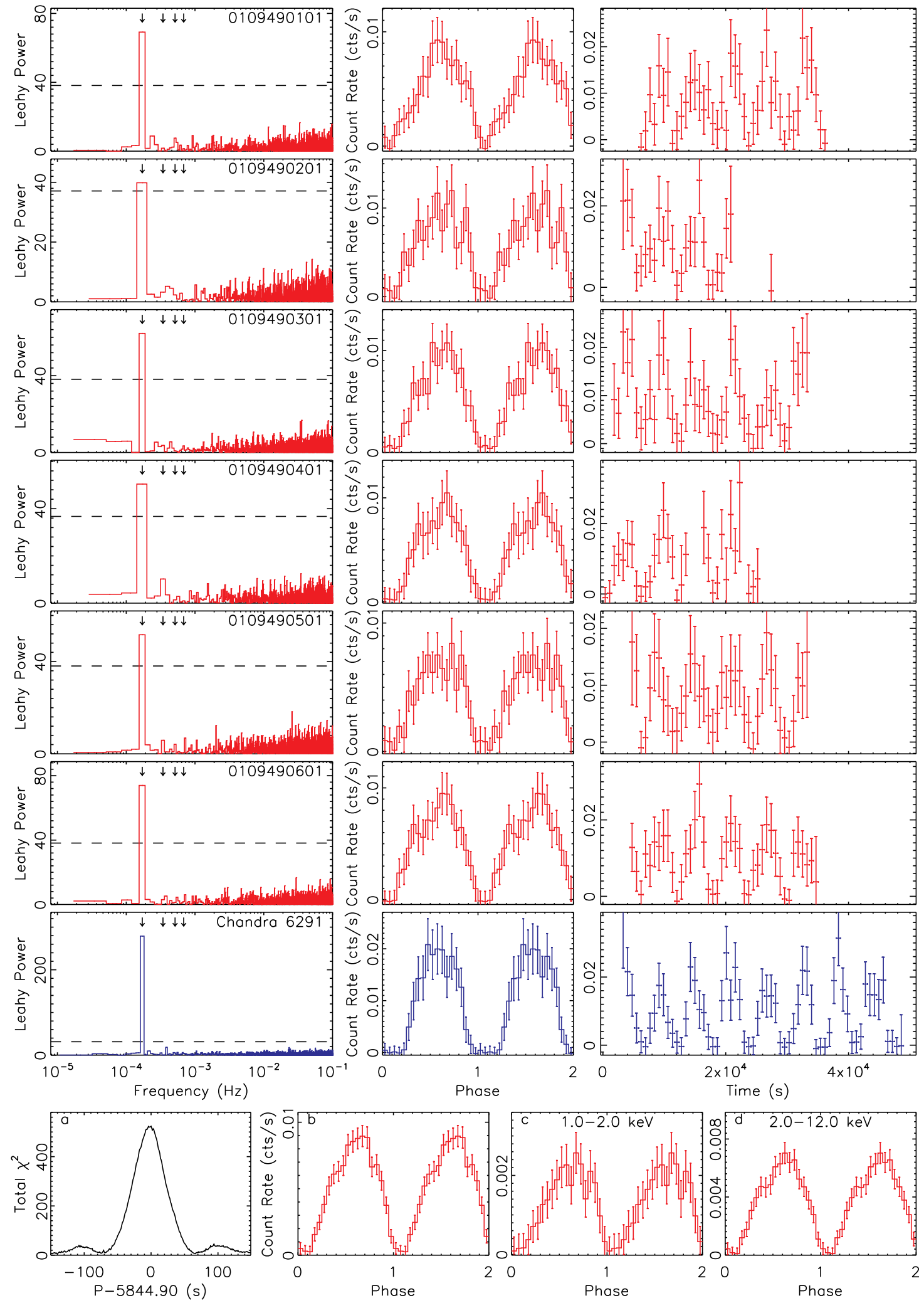

FIg. 4. - The timing properties of source \#150140. The panels in each row (except the botton one) show the Leahy power (left), the light curve folded at a period of $P_{0}=5844.90 \mathrm{~s}$ (middle), and the (unfolded) light curve (right) for each observation (1.0-12.0 keV). The arrows in the power plots mark the period of $P_{0}$ and harmonics $\left(P_{0} / 2, P_{0} / 3\right.$, and $\left.P_{0} / 4\right)$. The dashed lines indicate the $99.9 \%$ confidence detection level assuming blind search over all frequencies and Poisson noise as the underlying continuum. Only powers below $0.1 \mathrm{~Hz}$ are shown, and we see no powers above the $99.9 \%$ confidence level above $0.1 \mathrm{~Hz}$. The unfolded light curves are shifted in time to be aligned in phase. Panel (a) in the bottom row shows the total $\chi^{2}$ values from the fits with a constant to the $1.0-12.0 \mathrm{keV}$ light curves folded at various tentative periods using all six XMM-Newton observations 0109490101-601 (that is, the Chandra observation 6291 was not used). The 1.0-12.0 keV, 1.0-2.0 keV and 2.0-12.0 keV light curves folded at $P_{0}$ using also all six $X M M-N e w t o n$ observations are given in panels (b)-(d), respectively. 
TABLE 2

The Log of the XMM-Newton And Chandra Observations Analyzed

\begin{tabular}{|c|c|c|c|c|c|}
\hline SRCID & Obs. ID & Date & $\begin{array}{c}\text { off-axis angle } \\
\text { (arcmin) } \\
\mathrm{pn} / \mathrm{M} 1 / \mathrm{M} 2\end{array}$ & $\begin{array}{c}\text { Exposure } \\
(\mathrm{ks}) \\
\mathrm{pn} / \mathrm{M} 1 / \mathrm{M} 2\end{array}$ & $\begin{array}{c}r_{\mathrm{src}} \\
(\operatorname{arcsec})\end{array}$ \\
\hline 7204 & 0152020101 & 2003-06-20 & $8.1 / 7.3 / 6.6$ & $58.4 / 77.7 / 78.2$ & 15 \\
\hline \multirow{2}{*}{8085} & 0305860401 & $2005-05-22$ & $-/-/ 11.6$ & $-/-/ 34.7$ & 25 \\
\hline & 0112800201 & $2000-12-26$ & $1.5 / 11.6 / 12$. & $27.1 / 33.4 / 33.6$ & 15 \\
\hline 29251 & 0205230501 & $2004-11-23$ & $6.4 / 5.4 / 5.3$ & $12.5 / 15.5 / 15.5$ & 12 \\
\hline 65459 & 0306560301 & $2005-09-30$ & $2.7 / 1.7 / 1.8$ & $63.1 / 86.9 / 86.6$ & 12 \\
\hline \multirow{2}{*}{101335} & 0112830201 & $2000-12-22$ & $6.8 / 6.7 / 7.8$ & $50.8 / 58.9 / 58.9$ & 13 \\
\hline & 0402660201 & 2006-11-29 & $-/ 6.7 / 7.8$ & $-/ 29.3 / 28.8$ & 10 \\
\hline 101369 & 0112830201 & $2000-12-22$ & $7.0 / 6.9 / 5.9$ & $50.9 / 58.9 / 58.9$ & 14 \\
\hline 105130 & 0200340101 & $2004-06-03$ & $6.9 / 7.9 / 8.0$ & $60.4 / 75.7 / 76.7$ & 20 \\
\hline \multirow{3}{*}{107102} & 0145800101 & $2003-07-13$ & $7.5 / 7.1 / 8.1$ & $45.4 / 58.0 / 61.4$ & 25 \\
\hline & 0306630101 & 2005-12-13 & $6.8 /-/ 5.9$ & $54.8 /-/ 68.7$ & 25 \\
\hline & 0306630201 & 2005-12-17 & $6.8 /-/ 5.9$ & $80.8 /-/ 92.2$ & 25 \\
\hline \multirow{2}{*}{122979} & 0105261401 & $2002-07-27$ & $8.0 / 8.3 /-$ & $10.2 / 17.4 /-$ & 15 \\
\hline & 0105261701 & 2003-01-21 & $17.4 /-/-$ & $15.3 /-/-$ & 25 \\
\hline \multirow{4}{*}{142052} & 0203910101 & 2004-02-08 & $10.1 / 9.1 / 8.6$ & $5.9 / 8.7 / 8.7$ & 25 \\
\hline & 0402910101 & 2006-08-21 & $8.1 / 9.1 / 9.5$ & $38.8 / 4$ & 25 \\
\hline & 0410581901 & 2007-08-09 & $8.4 / 9.3 / 9.9$ & $13.2 / 1$ & 13 \\
\hline & 0560181101 & 2009-02-04 & $10.2 / 9.3 / 8.8$ & $48.5 / 57.1 / 57.2$ & 25 \\
\hline \multirow{7}{*}{150140} & 0109490101 & 2001-09-05 & $7.8 / 8.4 / 9.3$ & $27.4 / 32.9 / 32.9$ & 15 \\
\hline & 0109490201 & 2001-09-06 & $7.8 / 8.4 / 9.3$ & $15.9 / 20.1 / 20.4$ & 15 \\
\hline & 0109490301 & 2001-09-07 & $7.8 / 8.4 / 9.3$ & $28.4 / 34.0 / 34.1$ & 15 \\
\hline & 0109490401 & 2001-09-08 & $7.8 / 8.5 / 9.3$ & $17.9 / 30.5 / 30.7$ & 15 \\
\hline & 0109490501 & 2001-09-09 & $7.8 / 8.4 / 9.3$ & $25.4 / 30.7 / 30.7$ & 15 \\
\hline & 0109490601 & 2001-09-10 & $7.8 / 8.4 / 9.3$ & $27.1 / 32.5 / 32.6$ & 15 \\
\hline & $6291(\mathrm{CXO})$ & 2005-07-16 & 9.2 & 44 & 10 \\
\hline 165335 & 0149610401 & 2003-09-14 & $5.6 / 4.7 / 5.4$ & $27.3 / 32.0 / 32.0$ & 13 \\
\hline 174295 & 0200450301 & 2004-11-09 & $13.4 / 13.5 / 12.4$ & $-/ 21.4 / 21.4$ & 25 \\
\hline 183876 & 0311190101 & $2006-05-04$ & $8.2 / 8.2 / 9.2$ & $67.7 / 82.6 / 82.7$ & 12 \\
\hline 187831 & 0204610101 & $2005-04-30$ & $2.0 / 1.0 / 1.0$ & $63.0 / 77.7 / 78.0$ & 12 \\
\hline \multirow{24}{*}{222148} & 0505720201 & $2007-12-29$ & $-/ 13.1 / 12.7$ & $-/ 26.8 / 26.9$ & 20 \\
\hline & 0505720301 & 2008-01-08 & $-/ 13.1 / 12.9$ & $-/ 26.5 / 26.5$ & 20 \\
\hline & $0505720401^{\mathrm{a}}$ & 2008-01-18 & $-/ 13.1 / 13.0$ & $-/ 21.6 / 21.7$ & 20 \\
\hline & 0505720501 & $01-27$ & $-/ 13.1 /-$ & $-/ 20.0 /-$ & 20 \\
\hline & 050572 & 200 & $-/ 13.1 / 13.2$ & $-/ 21.2$ & 20 \\
\hline & 0551690201 & $12-30$ & $-/ 13.2 / 12.8$ & $-/ 21.2 / 21.2$ & 20 \\
\hline & 0551690301 & 2009-01-09 & $-/ 13.1 / 12.9$ & $-/ 21.1 / 21.1$ & 20 \\
\hline & $0551690401^{\mathrm{a}}$ & 2009-01-15 & $-/ 13.2 / 13.0$ & $-/ 6.1 / 5.8$ & 20 \\
\hline & 0551690501 & 2009-01-27 & $-/ 13.2 /-$ & $-/ 20.6 /-$ & 20 \\
\hline & $0551690601^{\mathrm{a}}$ & 2009-02-04 & $-/ 13.1 / 13.1$ & $-/ 10.9 / 10.6$ & 20 \\
\hline & 0600660201 & $2009-12-28$ & $-/ 13.1 / 12.7$ & $-/ 18.1 / 18.2$ & 20 \\
\hline & 0600660301 & 2010-01-07 & $-/ 13.1 / 12.9$ & $-/ 16.7 / 16.8$ & 20 \\
\hline & 0600660401 & 2010-01-15 & $-/ 13.1 / 13.0$ & $-/ 16.7 / 16.7$ & 20 \\
\hline & 0600660501 & 2010-01-25 & $-/ 13.1 /-$ & $-/ 19.1 /-$ & 20 \\
\hline & $0600660601^{\mathrm{a}}$ & 2010-02-02 & $-/ 13.1 / 13.1$ & $-/ 16.8 / 16.8$ & 20 \\
\hline & 0650560201 & $2010-12-26$ & $-/ 13.1 / 12.7$ & $-/ 24.1 / 24.3$ & 20 \\
\hline & 0650560301 & 2011-01-04 & $-/ 13.1 / 12.9$ & $-/ 32.4 / 32.7$ & 20 \\
\hline & 0650560401 & 2011-01-14 & $-/ 13.2 / 13.0$ & $-/ 18.8 / 19.4$ & 20 \\
\hline & 0650560501 & 2011-01-25 & $-/ 13.2 /-$ & $-/ 23.3 /-$ & 20 \\
\hline & 0650560601 & 2011-02-03 & $-/ 13.1 / 13.2$ & $-/ 23.3 / 23.2$ & 20 \\
\hline & 0672130101 & 2011-06-27 & $-/-/ 12.8$ & $-/-/ 99.3$ & 20 \\
\hline & 0672130601 & 2011-07-05 & $-/-/ 12.8$ & $-/-/ 91.5$ & 20 \\
\hline & 0672130701 & 2011-07-07 & $-/-/ 12.8$ & $-/-/ 89.9$ & 20 \\
\hline & $0672130501^{\mathrm{b}}$ & 2011-07-13 & $-/-/ 12.9$ & $-/-/ 42.2$ & 20 \\
\hline & 0405320801 & $2007-01-16$ & $2.6 / 1.7 / 2.4$ & $9.9 / 13.4 / 13.4$ & 13 \\
\hline & 0556300101 & $2008-05-19$ & $3.8 / 2.8 / 2.7$ & $18.7 / 30.0 / 30.6$ & $\overline{13}$ \\
\hline
\end{tabular}

Note. - For the Chandra observation 6291 (marked by "CXO"), the off-axis angle and the exposure refer to the ACIS detector.

${ }^{a}$ In these observations, the source is right at the edge of the field of view of the MOS2 camera.

b In this observation, the source is split by a CCD gap in the MOS2 camera

background spectrum was extracted from a large circular region, typically with a radius of $50^{\prime \prime}-100^{\prime \prime}$, near the source in each camera. The event selection criteria followed the default values in the pipeline (see Table 5 in Watson et al. 2009). We rebinned the spectra to have at least 20 counts in each bin so as to adopt the $\chi^{2}$ statistic for the spectral fits, but for some very faint detections, the spectra were not rebinned, and the fits were carried out using the $\mathrm{C}$ statistic. Because most of our data have low statistics, we fitted the spectra only with simple models: a powerlaw (PL, powerlaw in XSPEC Arnaud 1996)), a multicolor disk (MCD, diskbb in XSPEC), a single temperature blackbody (BB, bbodyrad in XSPEC), or their combinations. Sometimes, a Gaussian emission line (described by the gaussian model in XSPEC) is added when needed. All models included an interstellar medium absorption, described by the wabs model in XSPEC.

For sources that we found to show periodic modulations in the light curves from visual inspection, we created the Leahy power (Leahy et al. 1983) for each observation to confirm the presence of periodicity. We used pn light curves extracted from the source region and binned at the frame readout time (MOS1 was used for the observation 0105261401 of source \#122979 due to strong background flares in pn).

To constrain the values of their periods and examine the profiles of the modulation, we employed the epoch folding search technique. The folded backgroundsubtracted light curve for each camera is obtained by subtracting the folded light curve from the source region by the folded light curve from the background region (after being rescaled to the size of the source region). The total folded light curve for several cameras/observations are obtained by summing the folded background-subtracted light curves for individual cameras weighted by their total exposures (summed over all phase bins). All light curves were barycenter-corrected.

We also analyzed one Chandra observation of source \#150140 (Table 2) to check the persistence of its period. It was carried out using the imaging array of the AXAF CCD Imaging Spectrometer (ACIS; Bautz et al. 1998). We used the CIAO 4.4 package. The data were reprocessed to apply the latest calibration (CALDB 4.4.8). We used radii of $10^{\prime \prime}$ (Table 2) and $50^{\prime \prime}$ for the circular source and background regions, respectively.

\section{RESULTS AND DISCUSSION}

The long-term flux curves, light curves of bright detections, and sample spectral fits of our sources are shown in Figures 1 3, respectively. The spectral fit results are given in Table 3 .

\subsection{Periodic Hard Sources}

We discover signs of coherent pulsations from three hard X-ray sources, \#254026, \#150140 and \#122979. Source \#254026 was presented in Lin et al. (2013b), and in the following we focus on the latter two sources. Their X-ray spectra can be fitted with an absorbed PL, with photon indices $\Gamma_{\mathrm{PL}} \sim 1$ (Table 3). Neither of them have optical or IR counterparts found in the USNO-B1.0 Catalog (Monet et al. 2003) or the 2MASS Point Source Catalog (2MASS PSC, Cutri et al. 2003) within $4^{\prime \prime}$.

Source \#150140 appears on the outskirts of the young open cluster NGC 6231 (about $1.64 \mathrm{kpc}$ away and a few Myr old, Sana et al. 2006). There are six XMM-Newton observations spanning only five days in 2001 September, with $V_{\text {var } 14}=1.6$. The $0.3-10 \mathrm{keV}$ absorbed luminosity is about $7 \times 10^{31} \mathrm{erg} \mathrm{s}^{-1}$ if the source is in this cluster or about $2 \times 10^{33} \mathrm{erg} \mathrm{s}^{-1}$ if a source distance of $8.5 \mathrm{kpc}$ is assumed. There is a Chandra observation taken nearly four years later, with a similar spectrum (Table 3). The spectral fits indicate fairly strong absorption $\left(N_{\mathrm{H}} \sim 0.6 \times 10^{22}\right.$ 

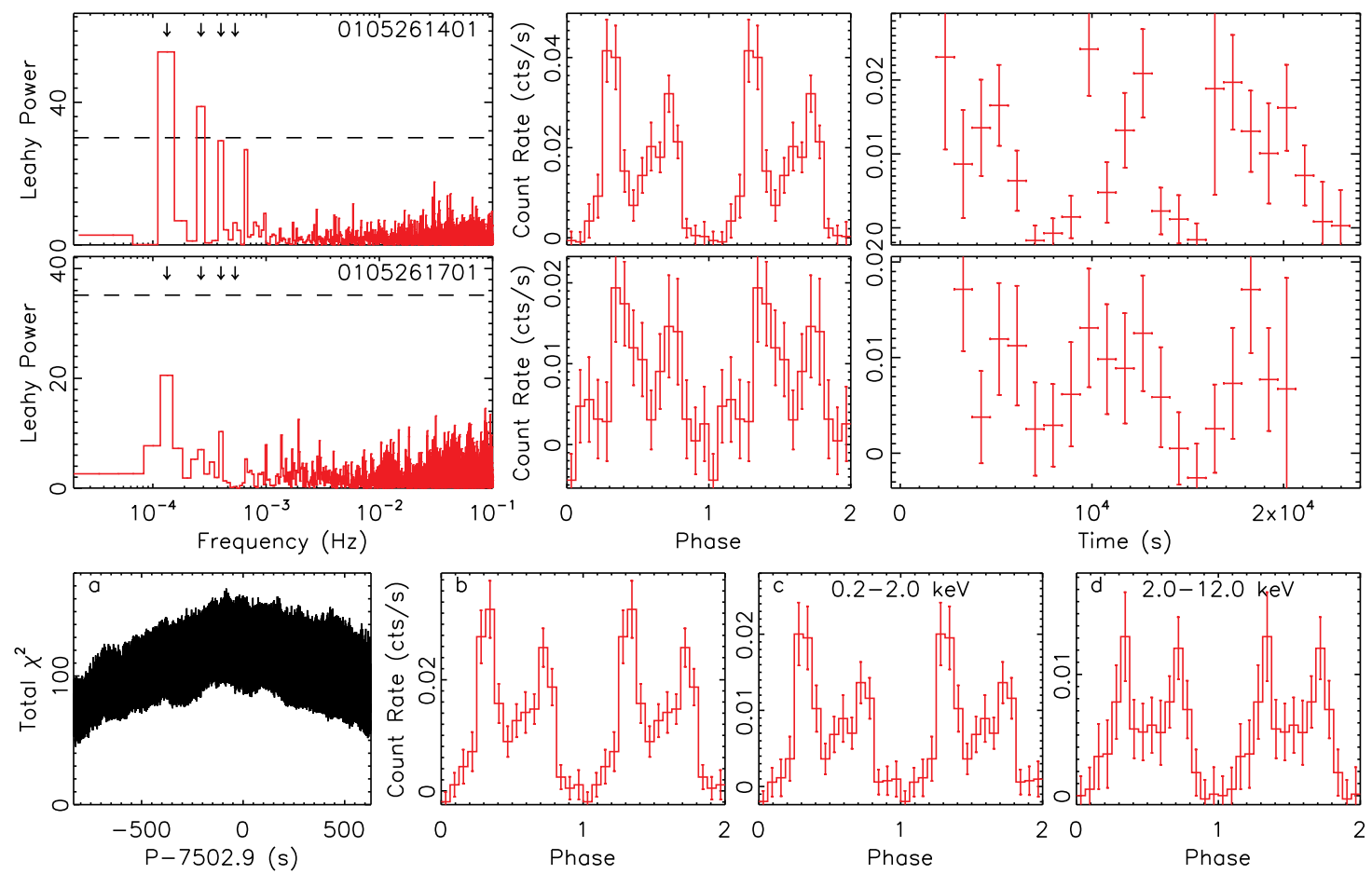

FIG. 5. - The timing properties of source \#122979, similar to Figure 4 The energy band 0.2-12.0 keV is used for all plots except panels (c)-(d). Both observations are combined to search for the periodicity (panel (a)) and plot the folded light curves in panels (b)-(d). The Leahy power and the (unfolded) light curve for observation 0105261401 are from MOS1.

$\mathrm{cm}^{-1}$ ) toward the source. Figure 4, which plots the timing properties of the source, shows that there are X-ray modulations at a period around 5845 s (i.e., $1.62 \mathrm{hr}$ ) in all the XMM-Newton and Chandra observations. In the search for the period using the epoch folding technique, we used only the XMM-Newton observations, as their time gap with Chandra observation is too large and adding the Chandra observation only results in many similar local maxima of the total $\chi^{2}$ near the period. The pulse profile shows only a single peak. The low phase has count rates consistent with zero but seems to last longer in the Chandra observation (for about $25 \%$ of the period) than in the XMM-Newton observations (for about $10 \%$ of the period). There is no significant variation of the pulse profile with energy. Considering its low X-ray luminosity and hard X-ray spectra, this source is probably a magnetic cataclysmic variable $(\mathrm{CV})$, i.e., a polar or a intermediate polar. With the period and the pulse profile resembling those of some short-period one-pole polars (e.g., V347 Pav and GG Leo, Ramsay et al. 2004), source \#150140 is most likely one such system. If it is really a $\mathrm{CV}$, it is probably not in NGC 6231, which seems too young to form a CV. This is also supported by the larger column density inferred from our spectral fitting than that of NGC $6231\left((2-3) \times 10^{21} \mathrm{~cm}^{-2}\right.$, Marggraf et al. 2004).

Source \#122979 has a Galactic latitude of $30.2^{\circ}$ and thus is probably nearby if it is in our Galaxy. It has two $X M M-N$-wton observations separated by about half a year, with $V_{\text {var14 }}=1.7$. The second observation has a very large off-axis angle of $17.4^{\prime}$, and the source is only in the FOV of pn. The maximum $0.3-10 \mathrm{keV}$ absorbed luminosity, from the first observation, is about $3 \times 10^{31}$ erg $\mathrm{s}^{-1}$, assuming a source distance of $1 \mathrm{kpc}$. We found a possible $\sim 2.1 \mathrm{hr}$ periodicity (Figure 5), which, however, needs to be confirmed with longer observations. The source might be a magnetic $\mathrm{CV}$, considering its probably low luminosity and hard X-ray spectra.

\subsection{Very Soft Sources}

We also found a handful of poorly studied very soft sources. Such sources have been observed in our Milky Way, the Magellanic Clouds, and nearby galaxies (for a recent review, see Di Stefano et al. 2010). They are often referred to as supersoft (BB temperature $k T_{\mathrm{BB}} \lesssim 0.1$ $\mathrm{keV})$ or quasisoft $\left(k T_{\mathrm{BB}} \lesssim 0.3 \mathrm{keV}\right)$ X-ray sources. They most probably represent a diverse group. If they are coincident with galactic nuclei, they could be ultrasoft AGNs or TDEs (e.g., Lin et al. 2011; Miniutti et al. 2013). If they are off-nuclear and have luminosities of $10^{35}-10^{38}$ erg $\mathrm{s}^{-1}$, they could be nuclear burning of material accreted by a WD (Greiner 2000), supernova remnants (e.g., Kong et al. 2003), central stars in planetary nebulae (e.g., Mereghetti et al. 2010), or hot stellar core remnants from tidal stripping of giants by SMBHs (Di Stefano et al. 2001). Those with luminosities below $10^{34}$ erg $\mathrm{s}^{-1}$ could be thermally cooling isolated NSs (e.g., Kaplan et al. 2002), NS X-ray binaries in quiescence (e.g., Webb \& Barret 2007), or WDs (e.g., WD 1254+223, which could be a binary (Bilíková et al. 2010)). Very soft sources with luminosities above the Eddington limit of WDs $\left(\sim 2 \times 10^{38}\right.$ erg $\mathrm{s}^{-1}$ ), such as ultraluminous off-nuclear X-ray sources (ULXs, $>10^{39} \mathrm{erg} \mathrm{s}^{-1}$ ), have two popular explanations: cool thermal disk emission from accreting intermediatemass BHs (IMBHs, $\sim 10^{2}-10^{5} M_{\odot}$, Miller et al. 2004; Kong \& Di Stefano 2005; Liu 2011) and optically thick outflows from supercritical accretion viewed at high inclinations (Mukai et al. 2003; King \& Pounds 2003; Poutanen et al. 2007; Feng \& Soria 2011). The spectral 

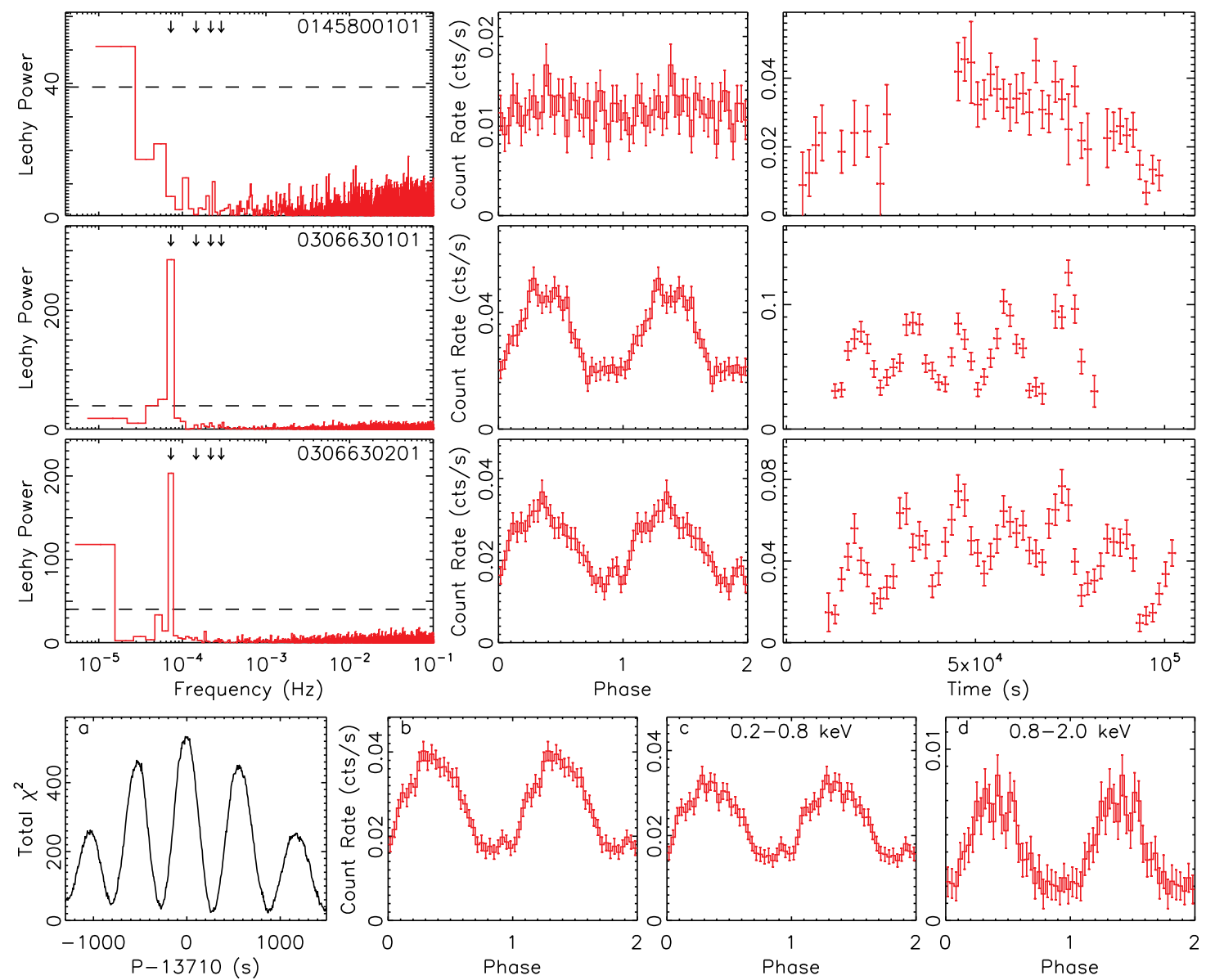

FIG. 6.- The timing properties of source \#107102, similar to Figure 4 The energy band 0.2-2.0 keV is used for all plots except panels (c) and (d). Only the two observations 0306630101-201 are combined to search for the periodicity (panel (a)) and plot the folded light curves in panels (b)-(d). The power of observation 0145800101 was calculated using only the second segment of data (after 40 ks in the light curve plot in the top right panel) because earlier data suffer from frequent strong background flares.

behavior in these two scenarios is expected to be different: the former should have the disk luminosity following the $L \propto T^{4}$ relation, and the latter should have the observed outflow luminosity decreasing slightly with increasing temperature (Poutanen et al. 2007). However, neither trend has been generally observed, and sometimes there is strong debate (e.g., Mukai et al. 2003; Kong \& Di Stefano 2005). We note that the supercritical accretion explanation for ultralumineous very soft sources might also need to involve IMBHs, instead of stellar-mass BHs. This is because edge-on viewing is required to have very soft spectra, by viewing the cool outflow only (not the central hot region), but at high inclinations, it is hard to achieve super-Eddington luminosities due to self-absorption and lack of beaming effect (Ohsuga et al. 2005).

We include seven very soft sources here, all with $k T_{\mathrm{BB}}<0.2 \mathrm{keV}$ (see below) and at high Galactic latitudes $\left(b=-22^{\circ}\right.$ for source $\# 65459$ and $|b| \gtrsim 60^{\circ}$ for the others). LWB12 selected out these sources for their low hardness ratios unseen in most AGNs (Table 11). To compare more with AGNs, we also calculated the strength of soft excess $R_{\text {exc }}$, defined as the ratio of unabsorbed $0.3-2 \mathrm{keV}$ fluxes in the cool and hard components Gierliński \& Done 2004), using the MCD+PL model (the $\mathrm{BB}+\mathrm{PL}$ model gave comparable results). Because our sources have no significant hard X-ray emis- sion, the PL component is generally not needed, in which case we fixed $\Gamma_{\mathrm{PL}}$ at a value of 2 often seen in AGNs (LWB12) and calculated the $90 \%$ lower limit of $R_{\text {exc }}$. Our sources have $R_{\text {exc }} \gtrsim 6$ (see below), while most AGNs have $R_{\text {exc }} \lesssim 1$ (Gierliński \& Done 2004).

\subsubsection{Two Sources Unassociated with Nearby Galaxies}

We first look at two sources, \#107102 and \#65459, which are not associated with any galaxy in the Third Reference Catalog (RC3, de Vaucouleurs et al. 1991). Source \#107102 is coincident with an optical source from the Sloan Digital Sky Survey (SDSS, Release \&4, Table 4), which appears slightly extended. Its Magellan optical spectrum taken by Ho et al. (2012) showed only narrow emission lines (no broad $\mathrm{H}_{\alpha}$ or $\mathrm{H}_{\beta}$ lines) that are consistent with a low-mass $\left(10^{5} M_{\odot}\right)$ type 2 Seyfert nucleus at a redshift of 0.11871 (a luminosity distance of 532 $\mathrm{Mpc})$. The source was detected in all its three $X M M$ Newton observations, with $V_{\text {var14 }}=2.1$. Their spectra are all very soft $\left(k T_{\mathrm{BB}} \sim 0.12-0.14 \mathrm{keV}\right.$ or $k T_{\mathrm{MCD}} \sim 0.15-0.18$ $\mathrm{keV}$, Table 3. Figure 3. $\left.R_{\text {exc }}>36\right)$. The $0.3-10 \mathrm{keV}$ absorbed luminosity reached a peak of $3.6 \times 10^{42} \mathrm{erg} \mathrm{s}^{-1}$ in the second observation (0306630101). Remarkably, the source showed large coherent oscillations at a period about $3.8 \mathrm{hr}$ in the two observations in 2005 December

\footnotetext{
${ }^{4}$ http://www.sdss3.org/
} 
but not in the observation in 2003 July (Figure 6). However, the minima and maxima of the light curves in the two observations in 2005 do not seem to be well in phase (Figure 6). Therefore we have probably detected a quasiperiodic oscialtion (QPO). The folded light curves in two energy bands $(0.2-0.8 \mathrm{keV}$ and $0.8-2 \mathrm{keV})$ in Figure 6 indicate that the modulation seems larger at higher energies. Considering the strong fast variability and little neutral absorption in X-rays, the lack of $\mathrm{H}_{\alpha}$ and $\mathrm{H}_{\beta}$ lines in the Magellan optical spectrum taken by Ho et al. (2012) should indicate the real absence of the broad line region, instead of being hidden (i.e., not a standard type 2 AGN), similar to GSN 069 (Miniutti et al. 2013). We have devoted a separate work (Lin et al. 2013a) to carry out detailed studies of the source, including formal calculation of the QPO significance by carefully modeling the red noise (the 99.9\% upper limits (dashed lines) in Figure 6 account for only the Poisson noise but not the red noise) and the spectral fits with more physical models. We refer to this work for more discussion on the nature of the source. We note that the source was also discovered independently by Terashima et al. (2012), who reported similar spectral and timing properties of the source as we show above. One main difference is that we show that the QPO is strong in both observations in 2005 December, not just in the first one as claimed by Terashima et al. (2012).

Source \#65459 was detected in one observation on 2005 September 30, but not in an earlier observation on 2003 December 19, with $V_{\text {var14 }}>3.2$ (Figure 1). The detection has $k T_{\mathrm{BB}}=0.11 \mathrm{keV}$ or $k T_{\mathrm{MCD}}=0.14 \mathrm{keV}$ (Table 3, Figure 3, $R_{\text {exc }}>12$ ). It shows some short-term variability (Figure 21). The source has no counterpart from the USNO-B1.0 Catalog or the 2MASS PSC (Table 11). It was not observed by the SDSS. Future deep optical imaging is needed to check whether it is associated with any galaxy to help to pin down its nature.

\subsubsection{Two Sources Near NGC 4151}

We next look at two sources, \#101369 and \#101335. They have angular separations from the center of the galaxy NGC 4151 of $\alpha=7.1^{\prime}$ and $6.6^{\prime}$, respectively. They were not considered to be associated with this galaxy by LWB12, because their ratios of the angular separation to the $D_{25}$ isophote elliptical radius $\alpha / R_{25}$ are 2.8 and 2.5, respectively, larger than the threshold value of 2 used to determine the association. Considering that NGC 4151 in fact has outer arms observed to extend out to $6^{\prime}$ (Pedlar et al. 1992), it is still possible that both sources are in this galaxy. Alternatively, they could be associated with distant galaxies, considering that both of them have SDSS optical counterpart candidates (Table (4).

Source \#101369 has eight XMM-Newton observations spanning nearly six years but was only detected in the first three observations which are continuous in time around 2000 December 22 and have very similar spectra, resulting in $V_{\text {var14 }}>5$ (Figure 1). The X-ray spectra are supersoft $\left(k T_{\mathrm{BB}} \sim 0.08 \mathrm{keV}\right.$, Table 3 , Figure 3, $R_{\text {exc }}=11$ ). The maximum $0.3-10 \mathrm{keV}$ absorbed luminosity is $1.3 \times 10^{39} \mathrm{erg} \mathrm{s}^{-1}$ if it is in NGC $4151(20.3$ Mpc, Liu \& Bregman 2005). The SDSS counterpart candidate of the source is slightly extended (the $r$-band Petrosian radius is $\sim 1^{\prime \prime} .5$ ) and has a photometric redshift of
$0.17 \pm 0.03$, corresponding to a luminosity distance of 790 Mpc. The maximum $0.3-10 \mathrm{keV}$ absorbed luminosity of the source is $2.0 \times 10^{42} \mathrm{erg} \mathrm{s}^{-1}$ using this distance.

Source \#101335 was detected in all its eight XMMNewton observations spanning nearly six years, with $V_{\text {var14 }}=2.6$ (Figure 11). We note that the source was at the CCD edge in some observations, which could affect the calculation of the long-term variability. The source was also detected in one Chandra detection in the CSC (Figure 11) and in several occasions by ROSAT HRI and PSPC in the early 1990s with similar fluxes (White et al. 1994), indicating the persistence of the source over nearly two decades. The fits with an absorbed PL to band count rates in $X M M$-Newton observations indicate that the source spectra were generally soft except the latest observation 0402660201 on 2006 November 29 (LWB12). We analyzed this observation and another observation 0112830201 on 2000 December 22 in detail (Tables 2 and 3. Figures 22 and 3). The second observation has a higher $\mathrm{S} / \mathrm{N}$, and it cannot be fitted well with a $\mathrm{BB}$, while the fit with a MCD is acceptable, with $k T_{\mathrm{MCD}}=0.21$ $\mathrm{keV}$ (Table 3, $R_{\text {exc }}>6$ ). The observation 0112830201 is fainter and shorter and has a low $\mathrm{S} / \mathrm{N}$, but the fit with a MCD requires a significantly higher temperature $k T_{\mathrm{MCD}}=0.59 \mathrm{keV}$, and a PL fit gives $\Gamma_{\mathrm{PL}}=2.1$, which probably indicates a state transition. If the source is in NGC 4151 , the $0.3-10 \mathrm{keV}$ absorbed luminosity reached $2.3 \times 10^{39} \mathrm{erg} \mathrm{s}^{-1}$ and $1.7 \times 10^{39} \mathrm{erg} \mathrm{s}^{-1}$ in the soft observation 0112830201 and the hard observation 0402660201 . The SDSS counterpart candidate of the source is faint and appears blue and point-like (Table 4).

Therefore, if they are in NGC 4151, both sources \#101369 and \#101335 would be very soft ULXs and could be due to cool thermal disk emission from accreting IMBHs or outflows from supercritical accretion. For source \#101335, there is possible state transition without large variation in luminosity, which could possibly be explained more easily with the former model. If the SDSS sources are their counterparts in this galaxy, these counterparts would have absolute $r$-band magnitudes about -12.0 and -9.9 , respectively, which are very bright and could be very massive star clusters. Alternatively, these SDSS sources are still their counterparts but are distant galaxies. Then sources \#101369 and \#101335 could be caused by nuclear activity. Considering its possible transient nature, sources \#101369 could be a TDE. For source \#101335, whose flux was relatively constant over nearly two decades, it could be an ultrasoft AGN similar to GSN 069 (Miniutti et al. 2013).

\subsubsection{Three Sources Associated with Nearby Galaxies}

The other three very soft sources that we analyzed are sources \#8085, \#183876, and \#187831, which LWB12 found to be likely within some RC3 galaxies. Source \#8085 was detected on the outskirts of NGC 300 $\left(\alpha / R_{25}=1.1\right.$ (Table 10) in all its four XMM-Newton observations spaning 4.9 years, but exhibiting large longterm variability $\left(V_{\mathrm{var} 14}=36\right.$, Figure 11). Clear spectral change was observed, with $k T_{\mathrm{BB}}$ varying from 0.07 to $0.12 \mathrm{keV}$ (Table 3, $R_{\text {exc }}>174$ ). Its maximum $0.3-10$ $\mathrm{keV}$ absorbed luminosity is $1.2 \times 10^{38} \mathrm{erg} \mathrm{s}^{-1}$, assuming a distance of $2 \mathrm{Mpc}$ (Freedman et al. 2001).

Source \#183876 was detected toward Seyfert 1 galaxy NGC $7314\left(\alpha / R_{25}=0.8\right)$ in two XMM-Newton observa- 
tions separated by 6 years, with no significant spectral variation observed $\left(V_{\text {var14 }}=1.2\right.$, Figure 11). The spectral fitting results of one observation (on 2006 May 4) are given in Table 3 and shown in Figure 3 . The spectrum has $k T_{\mathrm{BB}}=0.17 \mathrm{keV}$ or $k T_{\mathrm{MCD}}=0.21 \mathrm{keV}\left(R_{\mathrm{exc}}=11\right)$. Although we added a weak PL in the fit, this component can be due to contamination from the nucleus. There are also two detections in the CSC about one month after the second XMM-Newton observation, with comparable fluxes (Figure 11). Assuming a distance of 12.9 Mpc (Liu \& Bregman 2005), its 0.3-10 keV absorbed luminosity is about $8.6 \times 10^{38} \mathrm{erg} \mathrm{s}^{-1}$.

Source \#187831 was detected in the second of its three XMM-Newton observations of Seyfert 2 galaxy NGC $7582\left(\alpha / R_{25}=0.9, V_{\text {var14 }}>5.0\right.$, Figure 11). The detection has $k T_{\mathrm{BB}}=0.12 \mathrm{keV}$ or $k T_{\mathrm{MCD}}=0.14 \mathrm{keV}$ (Table 3. Figure 3. $R_{\text {exc }}>34$ ). The light curve exhibits significant variability (Figure 2). Assuming a distance of 17.6 Mpc (Liu \& Bregman 2005), we obtained a 0.3-10 keV absorbed luminosity of $1.1 \times 10^{39} \mathrm{erg} \mathrm{s}^{-1}$, making it a ULX. We note that this source is also close to another galaxy 6dFGS g2318191-422235 at a redshift of 0.05814 (Jones et al. 2009), at a separation of $4 . .6$ (corresponding to $5 \mathrm{kpc}$ ). Thus it is possible that source \#187831 is in this galaxy instead, which would increase its $0.3-10 \mathrm{keV}$ absorbed luminosity to be $2.3 \times 10^{41} \mathrm{erg} \mathrm{s}^{-1}$ (assuming a luminosity distance of $249.6 \mathrm{Mpc}$ ).

Sources \#183876 and \#187831 have maximum luminosities well above the Eddington limit of WDs $\left(\sim 2 \times 10^{38}\right.$ erg $\mathrm{s}^{-1}$ ) and are probably due to cool thermal disk emission from IMBHs or outflows from supercritical accretion. Source \#8085 could have a similar origin, instead of nuclear burning of material accreted by a WD, for its relatively high luminosity and BB temperature $(0.12$ $\mathrm{keV}$ ) in one observation. Among these three sources, only source \#8085 showed clear spectral variation, which could be used to differentiate between the thermal disk and outflow models. Both the BB and MCD fits suggest significant temperature variation at a relatively constant emission area (Table 3), which seems to favor the thermal disk model, but this should be confirmed with future high-quality observations.

\subsection{Sources with Large Long-term Variability}

\subsubsection{Off-nuclear Extragalactic Source Candidates}

We analyzed six sources that appear in nearby galaxies and show large long-term variability. Because of their high peak luminosities $\left(>10^{37}\right.$ erg $\mathrm{s}^{-1}$, see below) and the lack of very hard spectra expected for accretion-powered X-ray pulsars (LWB12), they are most likely accreting BHs or weakly magnetized NSs (they are mostly low-mass X-ray binaries) if they are indeed extragalactic (CVs have luminosities $<10^{33} \mathrm{erg} \mathrm{s}^{-1}$ when they are not in the supersoft phase (Muno et al. 2004)). The differentiation between accreting BHs and accreting weakly magnetized NSs is generally difficult, but hints can still be obtained in some cases. Observation of luminosities much higher than the Eddington limit for accreting NSs $\left(1.8 \times 10^{38} \mathrm{erg} \mathrm{s}^{-1}\right.$ assuming a $1.4-M_{\odot}$ NS and the pure hydrogen accreting material) and/or observation of soft spectra of $k T_{\mathrm{MCD}}<1 \mathrm{keV}$ at high luminosities will favor the accreting $\mathrm{BH}$ scenario (Remillard \& McClintock 2006), because accret- ing weakly magnetized NSs, even when they are in the soft state, seldom exhibit so soft spectra due to presence of the hot emission from the impact of accreting materials onto the NS surface (Done \& Gierliński 2003; Lin et al. 2007, 2009, 2010, 2012a). NS X-ray binaries can appear very soft only near quiescence $\left(<10^{34}\right.$ erg $\mathrm{s}^{-1}$, Webb \& Barret 2007).

We first look at four sources (\#7204, \#29251, \#105130 and \#241580) that are probably BH X-ray binaries. Source \#7204 was detected in one of nine observations of NGC $253\left(\alpha / R_{25}=0.74, V_{\text {var14 }}>23\right.$, Figure 11). The spectrum was fitted with an absorbed $\mathrm{PL}$ with $\Gamma_{\mathrm{PL}}=2.9$ by Barnard et al. (2008). We reanalyzed the data and obtained a consistent fit (Table 3 ). Considering the high value of $\Gamma_{\mathrm{PL}}$, we also fitted the spectra with a MCD plus a weak PL ( $\Gamma_{\mathrm{PL}}$ was fixed at a value of 2 due to its large uncertainty). The best-fitting disk temperature is about $0.5 \mathrm{keV}$, and the $0.3-10 \mathrm{keV}$ absorbed luminosity is $0.86 \times 10^{38} \mathrm{erg} \mathrm{s}^{-1}$ if a distance of $3.0 \mathrm{Mpc}$ is assumed (Liu 2011).

Source \#29251 was detected in two of fourteen observations of NGC $1313\left(\alpha / R_{25}=0.18, V_{\text {var14 }}>30\right.$, Figure 1). These two detections, separated by only 76 days, are probably from the same outburst. The fluxes and spectral shapes are similar in these two detections. We fitted the spectrum of one observation using the MCD model (Table 3. Figure 3) and obtained $k T_{\mathrm{MCD}}=0.8 \mathrm{keV}$ and a $0.3-10 \mathrm{keV}$ absorbed luminosity of $4.0 \times 10^{38} \mathrm{erg} \mathrm{s}^{-1}$, assuming a distance of 3.7 Mpc (Liu \& Bregman 2005). We note that an absorbed PL with $\Gamma_{\mathrm{PL}}=2.4$ can also fit the spectrum (Table 3). The source was also detected by ROSAT, with a slightly higher luminosity than we report here (Liu \& Bregman 2005), indicating the recurrent nature of the outbursts.

Source \#105130 was detected in three of five observations on the outskirts of NGC $4395\left(\alpha / R_{25}=1.65\right.$, $\left.V_{\text {var14 }}>65\right)$. We analyzed the brightest observation, taken on 2004 July 3, and found that the MCD model $\left(k T_{\mathrm{MCD}}=0.6 \mathrm{keV}\right)$ fitted the spectrum better than the $\mathrm{PL}$ and BB models, with the $\chi^{2}$ values decreased by 39 and 181 for 138 degrees of freedom, respectively (Table 3 . Figure 3). No significant short-term variation is seen in this observation (Figure 2). Assuming the source to be in NGC 4395 at a distance of $3.6 \mathrm{Mpc}$ (Liu \& Bregman 2005), we obtained the maximum $0.3-10 \mathrm{keV}$ absorbed luminosity of $1.9 \times 10^{38} \mathrm{erg} \mathrm{s}{ }^{-1}$. The source has two detections in the CSC (Figure 11), which, in addition to $X M M-N e w t o n$ observations, seem to suggest at least three outbursts within four years. The source was also detected in one ROSAT HRI observation 1RH702725N00 in $1996\left(2.3 \times 10^{38} \mathrm{erg} \mathrm{s}^{-1}\right.$, Liu \& Bregman 2005).

Source \#241580 was detected in two observations in 2008 May-June in the direction of NGC 4490 $\left(\alpha / R_{25}=0.87\right)$, but not in another observation in 2002 May $\left(V_{\text {var14 }}>16\right)$. The spectrum of the brightest observation can be fitted with an absorbed PL (Table 3 . Figure 3), with the corresponding $0.3-10 \mathrm{keV}$ absorbed luminosity of $7.6 \times 10^{38} \mathrm{erg} \mathrm{s}^{-1}$ if a distance of $7.8 \mathrm{Mpc}$ is assumed ( $\mathrm{Liu}$ 2011).

We see that sources \#7204, \#29251, and \#105130 have soft spectra with $k T_{\mathrm{MCD}} \lesssim 0.8 \mathrm{keV}$ and that sources \#29251, \#105130 and \#241580 have maximum 0.3-10 $\mathrm{keV}$ absorbed luminosities above the Eddington limit for 
accreting NSs. Therefore, these four sources are probably $\mathrm{BH}$ X-ray binaries.

We next look at two sources (\#222148 and \#222243) in M 31. Source \#222148 was detected in five of eighteen observations of M $31\left(\alpha / R_{25}=0.15, V_{\text {var14 }}>152\right)$. There are also eleven detections in the CSC. All these XMMNewton and Chandra detections spanned about 407 days from 2007 November to 2008 December (Figure 1). The outburst was discovered by Galache et al. (2007) from a 5ks Chandra observation on 2007 July 31, indicating that the outburst actually began earlier. To check whether the outburst is still ongoing, we analyzed 19 new observations that were not included in the 2XMMi-DR3 catalog but publicly available as of 2012 August (so covering data before 2011 July). The spectral fits using an absorbed PL for these 19 observations, in addition to the five detections included in the 2XMMi-DR3 catalog, are given in Table 3. The results show that the source has been X-ray bright for more than four years (this is also supported by Hofmann et al. (2013), who recently analyzed Chandra HRC observations of the source). The fluxes varied strongly at the beginning of the outburst and became fairly constant later. The spectra are consistently hard, with $\Gamma_{\mathrm{PL}} \sim 1.8$. One sample spectral fit is shown in Figure 3, using observation 0672130101, which has the most counts. The maximum $0.3-10 \mathrm{keV}$ absorbed luminosity is $6 \times 10^{37} \mathrm{erg} \mathrm{s}^{-1}$, and the average is around $4 \times 10^{37} \mathrm{erg} \mathrm{s}^{-1}$ (assuming a distance of 780 kpc, Stanek \& Garnavich 1998). Although we found no optical and IR counterparts from the USNO-B1.0 Catalog and 2MASS PSC, there is an optical source LGGS J004211.09+410431.0 in Massey et al. (2007) at a separation of 1 ." 8 , which has a $V$-band magnitude of $22.4 \pm 0.1$ and colors of $B-V=1.4 \pm 0.4, V-R=0.6 \pm 0.1$ and $R-I=1.0 \pm 0.1$ and is probably a late-type dwarf star.

There have been some X-ray binaries known to remain X-ray bright most of the time since they were discovered to begin the outburst, e.g., the $\mathrm{BH}$ X-ray binary candidates Swift J1753.5-0127 (since 2005 May, Soleri et al. 2010) and GRS 1915+105 (since $1992 \mathrm{Au}-$ gust, McClintock \& Remillard 2006) and the accretionpowered millisecond X-ray pulsar HETE J1900.1-2455 (since 2005 June, Galloway et al. 2007). Swift J1753.50127 is essentially in the hard state, with $2-10 \mathrm{keV}$ luminosity mostly around $5 \times 10^{36} \mathrm{erg} \mathrm{s}^{-1}$ Soleri et al. 2010). HETE J1900.1-2455 has a similar mean luminosity $\left(4.4 \times 10^{36} \mathrm{erg} \mathrm{s}^{-1}\right.$ in $2.5-25 \mathrm{keV}$, Galloway et al. 2008) and is mostly (but not always) in the hard state based on the hardness ratios that we calculated from the RXTE monitoring pointed observations (following a method as described in Lin et al. (2007)). GRS 1915+105 exhibits complicate behavior and has shown several states (including both the hard and soft/thermal states). If source \#222148 is a BH X-ray binary, it should be in the hard state. To check whether the source could be an accreting weakly magnetized NS in the hard state, in which a BB component is normally present and contributes a few percent (but $<10 \%$ ) of the luminosity (Lin et al. 2007, 2010), we added a BB component in the fit to the observation 0672130101 . We found that the spectral fit was not significantly improved (the $\chi^{2}$ decreased by 2.6 from 63.0 while the degrees of freedom decreased by 2 from 74 ), with the BB flux estimated to be $<20 \%$ of the total $(0.3-10 \mathrm{keV}$, absorbed) at a $90 \%$-confidence level. Thus, although we do not need the $\mathrm{BB}$ in the fits, we cannot exclude its presence at the level expected for accreting weakly magnetized NSs in the hard state. However, the luminosity of accreting weakly magnetized NSs in the hard state is normally lower than $10 \%$ of the Eddington limit (Lin et al. 2007, 2010; Galloway et al. 2008; Hartman et al. 2009), while source \#222148 is slightly brighter (by a factor of 2 or more if the luminosity is estimated over a broader energy band). We note that we cannot exclude the possibility that the source is in the soft state of an accreting weakly magnetized NS based on the spectral fit. To demonstrate this, we also fitted the spectrum of observation 0672130101 with a model of $\mathrm{MCD}+\mathrm{BB}$, which are often used to fit the soft state of an accreting weakly magnetized NS (Mitsuda et al. 1984; Lin et al. 2007, 2009, 2010, 2012a) and obtained a similar quality of the fit as that using an absorbed PL (Table 3). This is because the soft-state spectra of accreting weakly magnetized NSs can still appear hard below about $7 \mathrm{keV}$. Future broad band observations with observatories such as NuSTAR can break such a model degeneracy easily.

Source \#222243 was detected in three of nineteen observations of M 31. It is only $\alpha=1.5^{\prime}\left(\alpha / R_{25}=0.02\right)$ away from the center of M 31. LWB12 measured $V_{\text {var14 }}>6$ and $V_{\operatorname{var}}(0.2-12.0 \mathrm{keV})>10$, but these variation factors were most likely underestimated due to the bright diffuse emission in the galaxy center. The CSC indicates that the source was also detected in nine Chandra observations from 2007 March to 2008 December, with 0.1-10 $\mathrm{keV}$ fluxes about $10^{-14} \mathrm{erg} \mathrm{s}^{-1} \mathrm{~cm}^{-2}$. In fact, there are many more Chandra observations of this source, indicating that the source is generally faint for 12 years (Barnard et al. 2012; Hofmann et al. 2013). In comparison, the three XMM-Newton observations in which the source was detected were made at the beginning of 2007 January, with $0.2-12.0 \mathrm{keV}$ fluxes in the range of (1.4$12.2) \times 10^{-13} \mathrm{erg} \mathrm{s}^{-1} \mathrm{~cm}^{-2}$. Their spectra are all hard. We analyzed the brightest observation 0405320801 and found clear residuals when we fitted the spectrum with a single component model (i.e., an absorbed MCD, BB, or PL). We obtained an acceptable fit with a MCD+BB model (Table 3 and Figure 3), indicating that the source might be an accreting weakly magnetized NS in the soft state (see discussion above for source \#222148). The $0.3-10 \mathrm{keV}$ absorbed luminosity is $6.8 \times 10^{37} \mathrm{erg} \mathrm{s}^{-1}$ (assuming a distance of $780 \mathrm{kpc}$, Stanek \& Garnavich 1998). The source is probably in the globular cluster candidate BH16 (i.e., PB-in7, Galleti et al. 2004; Barnard et al. 2012).

\subsubsection{Galactic Source Candidates}

Sources \#142052, \#165335 and \#174295 are at low Galactic latitudes $\left(|b|<3^{\circ}\right)$ and have Galactic longitudes of about $327^{\circ}, 11^{\circ}$, and $80^{\circ}$, respectively. They are highly variable $\left(V_{\text {var14 }}>20\right.$, Figure 1, Table 1). Their X-ray spectra are generally hard and can be fitted with an absorbed PL (when detected, Table 3, Figure 3). They all have optical and IR counterparts found within $1.3^{\prime \prime}$ from the USNO-B1.0 Catalog and the 2MASS PSC. LWB12 classified them as compact object systems based on high X-ray-to-IR flux ratios, lack of flares, and large 
variability.

Source \#142052 was detected in all four XMMNewton observations and one Chandra observation (ObsID: 7287, from the CSC) spanning five years, with $V_{\text {var14 }}=23$ (Figure 11). It is probably AX J1549.8-5416, which was detected in the $A S C A$ Galactic Plane Survey (Sugizaki et al. 2001). Thus the source is probably persistent but highly variable. In the spectral fits to two long XMM-Newton observations (0402910101 and 0560181101), a Gaussian Fe line was detected above $3 \sigma$ (based on the normalization), with an equivalent width (EW) of about $1.0 \mathrm{keV}$ (Table 3). The source peak luminosity is in the range between about $2 \times 10^{32}$ and $10^{34} \mathrm{erg}$ $\mathrm{s}^{-1}(0.3-10 \mathrm{keV}$, absorbed) if the source distance is between $1-8.5 \mathrm{kpc}$. Its optical counterpart is probably NSV 20407 , a variable star with the $B$-band magnitude ranging from $<18$ to 16.7 (Samus et al. 2009) ), supporting to identify it as a CV. Further considering that the magnetic CVs often exhibit the Fe emission line (Ezuka \& Ishida 1999), source \#142052 is probably one such system.

Source \#165335 was detected in one of its two XMMNewton observations $\left(V_{\text {var14 }}>25\right)$. It was also detected in a Chandra observation in 2006 (ObsID: 6405, from the CSC) and is source 23 in Pravdo et al. (2009) in the direction of the MRR 32 X-ray cluster. The flux in this Chandra observation is very low, resulting in a 0.5-7 keV flux variation factor of 167, compared with the XMM-Newton detection. The $0.3-10 \mathrm{keV}$ absorbed luminosity in the $X M M-N e w t o n$ detection is within the range of $8 \times 10^{31}$ to $6 \times 10^{33} \mathrm{erg} \mathrm{s}^{-1}$ if the source distance is between $1-8.5 \mathrm{kpc}$. Considering that it appears in a star forming region, the bright XMM-Newton observation, which last $32 \mathrm{ks}$ and showed no obvious variability, might be due to an extremely long (probably several days) stellar X-ray flare. However, stellar X-ray flares with durations of days and flux increasing factors of more than one hundred are rarely seen. Flares lasting for days or more have been detected before from RS CVn stars (e.g., Kuerster \& Schmitt 1996; Endl et al. 1997, and references therein), but they have flux increasing factors of only a few tens. LWB12 reported several flares (e.g., sources \#64367 and \#146561) with flux increasing factors of more than one hundred, but they remained bright for only a few ks. If it is not a star, it is most probably a CV or a very faint X-ray transient (VFXT) with a $\mathrm{BH}$ or NS as the accretor (Wijnands et al. 2006), considering the very low peak luminosity of the source. VFXTs are transient sources with peak outburst luminosities between $10^{34}$ and $\lesssim 10^{36} \mathrm{erg} \mathrm{s}^{-1}$. The lower limit of $10^{34}$ erg $\mathrm{s}^{-1}$ is adopted in the definition so that they are not CVs but probably accreting BHs or NSs. It is not clear whether they can in fact have fainter outbursts. Most known VFXTs were detected toward the Galactic center from the intensive monitoring of the Galactic center by XMM-Newton and Chandra (Sakano et al. 2005; Muno et al.|2005; Wijnands et al.|2006). Our knowledge on this class of objects is still very limited.

Source \#174295 was securely detected in an observation on 2004 November 9, with a hard and highly absorbed spectrum as indicated by the fits with an absorbed PL (Table [3. Figure [3), but it was hardly detected in the observations ten days before and ten days after it, indicating a short outburst. The source has a candidate SDSS point-like counterpart (Table 4), which appears very red, probably caused by strong absorption. The maximum $0.3-10 \mathrm{keV}$ absorbed luminosity would be between $2 \times 10^{32}$ and $10^{34} \mathrm{erg} \mathrm{s}^{-1}$ if the source distance is within 1-8.5 kpc. Similar to our discussion for source \#165335 above, this source might be a CV, a VFXT with a $\mathrm{BH}$ or NS as the accretor, or a star with an extremely energetic stellar flare.

\section{CONCLUSIONS}

We have studied 18 sources from the 2XMMi-DR3 catalog that were poorly studied in the literature but show interesting properties of periodicity, very soft spectra and/or large long-term variability in X-rays in order to investigate their nature. Our findings can be summarized as follows:

1. Two sources have been persistently detected with hard spectra $\left(\Gamma_{\mathrm{PL}} \sim 1\right)$. One of them (source \#150140) was detected on the outskirts of the young open cluster NGC 6231 and showed a $1.62 \mathrm{hr}$ periodicity in six XMM-Newton observations and one Chandra observation separated by nearly four years. There is also a $\sim 2.1 \mathrm{hr}$ period candidate in the other source (\#122979), but it needs to be confirmed with future long observations. Both sources are good magnetic CV candidates.

2. Seven very soft sources $\left(k T_{\mathrm{BB}}<0.2 \mathrm{keV}\right)$ are probably in other galaxies, which would imply the luminosities ranging from about $10^{38}$ to $10^{42} \mathrm{erg} \mathrm{s}^{-1}$. Some of them might be coincident with galactic nuclei and best explained as ultrasoft AGNs or TDEs, while the others are probably off-nuclear and could be cool thermal disk emission from accreting IMBHs or edge-on viewing of optically thick cool outflows from supercritical accretion. One source (\#107102) is an AGN candidate and showed an intermittent QPO at about $3.8 \mathrm{hr}$, i.e., in two observations in 2005 December but not in one observation in 2003 July.

3. Six highly variable sources with spectra harder than the above very soft sources appear in nearby RC3 galaxies and probably have luminosities above $10^{37} \mathrm{erg} \mathrm{s}^{-1}$, making them great candidates for extragalactic X-ray binaries. One source (\#222148, in M 31) has remained X-ray bright and hard in $0.3-10 \mathrm{keV}$ since it was discovered to enter an outburst on 2007 July 31 and thus is probably a newborn persistent source.

4. Three highly variable hard sources appear at low galactic latitudes and have maximum luminosities below about $10^{34} \mathrm{erg} \mathrm{s}^{-1}$ if they are in our Galaxy. They could be CVs, VFXTs with a BH or NS as the accretor, or stars with extremely strong flares.

Although most of our sources still require future optical spectroscopic observations and/or long-term X-ray monitoring to finally pin down their nature, it is clear that our sample contains a variety of objects with all kinds of spectral behavior. Some of them belong to rare classes that still have relatively small numbers of sources 
known, such as very soft X-ray sources, very faint transient and new-born persistent sources, and our discovery of new objects for these classes is thus important for our understanding of them. We found their properties mostly consistent with previous studies, but some new results were also obtained. Focusing on the seven very soft X-ray sources, we found the existence of both supersoft and quasisoft X-ray sources and the possibility that some of them are the same class of objects because supersoft and quasisoft spectra can be observed in a single source (see source \#8085), consistent with previous studies (e.g., Kong \& Di Stefano 2005). However, we also observed some evidence that they displayed various kinds of spectral evolution behavior (being transient or persistent with either small or large long-term variability and displaying state transition) often seen in X-ray binaries. It is not clear whether this is completely because our sample might include several classes of objects. Our discovery of some unique objects is also significant, especially source \#107102, as an AGN candidate with very soft spectra and a strong QPO in X-rays (Lin et al. 2013a). There is only one other AGN known to show similarly weak hard X-ray emission (GSN 069, Miniutti et al.|2013) and only two other galactic nuclei with QPOs significantly detected (RE J1034+396, Gierliński et al. 2008; Swift J164449.3+573451, Reis et al. 2012).

Acknowledgments: We thank the anonymous referee for the helpful comments. We acknowledge the use of public data from the Chandra and XMM-Newton data archives, the 2XMM Serendipitous Source Catalog, constructed by the XMM-Newton Survey Science Center on behalf of ESA, and the Chandra Source Catalog, provided by the Chandra X-ray Center as part of the Chandra Data Archive.

\section{REFERENCES}

Arnaud, K. A. 1996, in Astronomical Society of the Pacific Conference Series, Vol. 101, Astronomical Data Analysis Software and Systems V, ed. G. H. Jacoby \& J. Barnes, 17-+

Barnard, R., Garcia, M., \& Murray, S. S. 2012, ApJ, 757, 40

Barnard, R., Greening, L. S., \& Kolb, U. 2008, MNRAS, 388, 849

Bautz, M. W., Pivovaroff, M., Baganoff, F., et al. 1998, in Society of Photo-Optical Instrumentation Engineers (SPIE) Conference Series, Vol. 3444, Society of Photo-Optical Instrumentation Engineers (SPIE) Conference Series, ed. R. B. Hoover \& A. B. Walker, 210-224

Bilíková, J., Chu, Y., Gruendl, R. A., \& Maddox, L. A. 2010, AJ, 140,1433

Cutri, R. M., Skrutskie, M. F., van Dyk, S., et al. 2003, VizieR Online Data Catalog, 2246, 0

de Vaucouleurs, G., de Vaucouleurs, A., Corwin, Jr., H. G., et al. 1991, Third Reference Catalogue of Bright Galaxies, ed. de Vaucouleurs, G., de Vaucouleurs, A., Corwin, H. G., Jr., Buta, R. J., Paturel, G., \& Fouque, P.

Di Stefano, R., Greiner, J., Murray, S., \& Garcia, M. 2001, ApJ, 551, L37

Di Stefano, R., Kong, A., \& Primini, F. A. 2010, New A Rev., 54, 72

Done, C. \& Gierliński, M. 2003, MNRAS, 342, 1041

Endl, M., Strassmeier, K. G., \& Kurster, M. 1997, A\&A, 328, 565

Evans, I. N., Primini, F. A., Glotfelty, K. J., et al. 2010, ApJS, 189,37

Ezuka, H. \& Ishida, M. 1999, ApJS, 120, 277

Feng, H. \& Soria, R. 2011, New A Rev., 55, 166

Freedman, W. L., Madore, B. F., Gibson, B. K., et al. 2001, ApJ, 553,47

Galache, J. L., Garcia, M. R., Steeghs, D., et al. 2007, The Astronomer's Telegram, 1171, 1

Galleti, S., Federici, L., Bellazzini, M., Fusi Pecci, F., \& Macrina, S. 2004, A\&A, 416, 917

Galloway, D. K., Morgan, E. H., \& Chakrabarty, D. 2008, in American Institute of Physics Conference Series, Vol. 1068, American Institute of Physics Conference Series, ed.

R. Wijnands, D. Altamirano, P. Soleri, N. Degenaar, N. Rea, P. Casella, A. Patruno, \& M. Linares, 55-62

Galloway, D. K., Morgan, E. H., Krauss, M. I., Kaaret, P., \& Chakrabarty, D. 2007, ApJ, 654, L73

Gierliński, M. \& Done, C. 2004, MNRAS, 349, L7

Gierliński, M., Middleton, M., Ward, M., \& Done, C. 2008, Nature, 455, 369

Greiner, J. 2000, New Astronomy, 5, 137

Hartman, J. M., Watts, A. L., \& Chakrabarty, D. 2009, ApJ, 697, 2102

Ho, L. C., Kim, M., \& Terashima, Y. 2012, ApJ, 759, L16

Hofmann, F., Pietsch, W., Henze, M., et al. 2013, A\&A, 555, A65

Jones, D. H., Read, M. A., Saunders, W., et al. 2009, MNRAS, 399,683

Kaplan, D. L., van Kerkwijk, M. H., \& Anderson, J. 2002, ApJ, 571,447
King, A. R. \& Pounds, K. A. 2003, MNRAS, 345, 657

Kong, A. K. H. \& Di Stefano, R. 2005, ApJ, 632, L107

Kong, A. K. H., Sjouwerman, L. O., Williams, B. F., Garcia, M. R., \& Dickel, J. R. 2003, ApJ, 590, L21

Kuerster, M. \& Schmitt, J. H. M. M. 1996, A\&A, 311, 211

Leahy, D. A., Darbro, W., Elsner, R. F., et al. 1983, ApJ, 266, 160

Lidskii, V. V. \& Ozernoi, L. M. 1979, Soviet Astronomy Letters, 5,16

Lin, D., Carrasco, E. R., Grupe, D., et al. 2011, ApJ, 738, 52

Lin, D., Irwin, J. A., Godet, O., Webb, N. A., \& Barret, D. 2013a, ApJ, 776, L10

Lin, D., Remillard, R. A., \& Homan, J. 2007, ApJ, 667, 1073

—. 2009, ApJ, 696, 1257

-. 2010, ApJ, 719, 1350

Lin, D., Remillard, R. A., Homan, J., \& Barret, D. 2012a, ApJ, 756,34

Lin, D., Webb, N. A., \& Barret, D. 2012b, ApJ, 756, 27

-. 2013b, ApJ, 766, 29

Liu, J. 2011, ApJS, 192, 10

Liu, J.-F. \& Bregman, J. N. 2005, ApJS, 157, 59

Marggraf, O., Bluhm, H., \& de Boer, K. S. 2004, A\&A, 416, 251

Massey, P., Olsen, K. A. G., Hodge, P. W., et al. 2007, AJ, 133, 2393

McClintock, J. E. \& Remillard, R. A. 2006, Compact Stellar X-ray Sources, ed. W. Lewin and M. van der Klis (Cambridge: Cambridge Univ. Press), 157-213

Mereghetti, S., Krachmalnicoff, N., La Palombara, N., et al. 2010, A\&A, 519, A42

Miller, J. M., Fabian, A. C., \& Miller, M. C. 2004, ApJ, 614, L117

Miniutti, G., Saxton, R. D., Rodríguez-Pascual, P. M., et al. 2013, MNRAS, 433, 1764

Mitsuda, K., Inoue, H., Koyama, K., et al. 1984, PASJ, 36, 741

Monet, D. G., Levine, S. E., Canzian, B., et al. 2003, AJ, 125, 984

Mukai, K., Pence, W. D., Snowden, S. L., \& Kuntz, K. D. 2003, ApJ, 582, 184

Muno, M. P., Arabadjis, J. S., Baganoff, F. K., et al. 2004, ApJ, 613,1179

Muno, M. P., Pfahl, E., Baganoff, F. K., et al. 2005, ApJ, 622, L113

Ohsuga, K., Mori, M., Nakamoto, T., \& Mineshige, S. 2005, ApJ, 628,368

Pedlar, A., Howley, P., Axon, D. J., \& Unger, S. W. 1992, MNRAS, 259, 369

Poutanen, J., Lipunova, G., Fabrika, S., Butkevich, A. G., \& Abolmasov, P. 2007, MNRAS, 377, 1187

Pravdo, S. H., Tsuboi, Y., Suzuki, Y., Thompson, T. J., \& Rebull, L. 2009, ApJ, 690, 850

Ramsay, G., Cropper, M., Mason, K. O., Córdova, F. A., \& Priedhorsky, W. 2004, MNRAS, 347, 95

Rees, M. J. 1988, Nature, 333, 523

Reis, R. C., Miller, J. M., Reynolds, M. T., et al. 2012, Science, 337,949

Remillard, R. A. \& McClintock, J. E. 2006, ARA\&A, 44, 49 
Sakano, M., Warwick, R. S., Decourchelle, A., \& Wang, Q. D. 2005, MNRAS, 357, 1211

Samus, N. N., Durlevich, O. V., \& et al. 2009, VizieR Online Data Catalog, 1, 2025

Sana, H., Gosset, E., Rauw, G., Sung, H., \& Vreux, J.-M. 2006, A\&A, 454, 1047

Soleri, P., Fender, R., Tudose, V., et al. 2010, MNRAS, 406, 1471

Stanek, K. Z. \& Garnavich, P. M. 1998, ApJ, 503, L131

Sugizaki, M., Mitsuda, K., Kaneda, H., et al. 2001, ApJS, 134, 77

Terashima, Y., Kamizasa, N., Awaki, H., Kubota, A., \& Ueda, Y. 2012, ApJ, 752, 154
Watson, M. G., Schröder, A. C., Fyfe, D., et al. 2009, A\&A, 493, 339

Webb, N. A. \& Barret, D. 2007, ApJ, 671, 727

White, N. E., Giommi, P., \& Angelini, L. 1994, IAU Circ., 6100, 1 Wijnands, R., in't Zand, J. J. M., Rupen, M., et al. 2006, A\&A, 449,1117 
TABLE 3

Spectral fit Results

\begin{tabular}{|c|c|c|c|c|c|c|c|c|c|c|c|}
\hline $\begin{array}{r}\text { SRCID } \\
\text { (1) }\end{array}$ & $\begin{array}{c}\text { Obs ID } \\
\text { (2) }\end{array}$ & $\begin{array}{c}N_{\mathrm{H}} \\
\left(10^{22} \mathrm{~cm}^{-2}\right) \\
(3)\end{array}$ & $\begin{array}{c}k T_{\mathrm{MCD}} \\
(\mathrm{keV}) \\
(4)\end{array}$ & $\begin{array}{c}N_{\mathrm{MCD}} \\
(5)\end{array}$ & $\begin{array}{c}k T_{\mathrm{BB}} \\
(\mathrm{keV}) \\
(6)\end{array}$ & $\begin{array}{l}N_{\mathrm{BB}} \\
(7)\end{array}$ & $\begin{array}{c}\Gamma_{\mathrm{PL}} \\
(8)\end{array}$ & $\begin{array}{c}N_{\mathrm{PL}} \\
\left(10^{-6}\right) \\
(9)\end{array}$ & $\begin{array}{c}\chi_{\nu}^{2}(\nu)^{\mathrm{a}} \\
(10)\end{array}$ & $\begin{array}{c}F_{\text {abs }} \\
\left(10^{-13} \text { erg }\right. \\
(11)\end{array}$ & $\begin{array}{c}F_{\text {unabs }} \\
\left.\mathrm{s}^{-1} \mathrm{~cm}^{-2}\right) \\
(12)\end{array}$ \\
\hline \multirow{2}{*}{7204} & \multirow{2}{*}{0152020101} & $0.01_{-0.01}^{+0.02}$ & $0.47_{-0.05}^{+0.04}$ & $0.06_{-0.02}^{+0.04}$ & $\cdots$ & $\cdots$ & 2.0 & $4_{-1}^{+3}$ & $0.91(101)$ & $0.80_{-0.06}^{+0.06}$ & $0.82_{-0.08}^{+0.10}$ \\
\hline & & $0.17_{-0.03}^{+0.03}$ & $\cdots$ & $\cdots$ & $\cdots$ & $\cdots$ & $3.1_{-0.2}^{+0.2}$ & $39_{-4}^{+5}$ & $1.04(102)$ & $0.79_{-0.05}^{+0.05}$ & $2.1_{-0.3}^{+0.4}$ \\
\hline \multirow{4}{*}{8085} & \multirow[t]{2}{*}{$0305860401^{b}$} & $0.13_{-0.07}^{+0.10}$ & $\cdots$ & $\cdots$ & $0.12_{-0.02}^{+0.02}$ & $\begin{array}{l}431_{-286}^{+1275} \\
\end{array}$ & $\cdots$ & $\cdots$ & $1.22(20)$ & $2.6_{-0.2}^{+0.2}$ & $7_{-3}^{+8}$ \\
\hline & & $0.20_{-0.08}^{+0.11}$ & $0.14_{-0.02}^{+0.02}$ & $432_{-322}^{+1874}$ & $\cdots$ & $\cdots$ & $\cdots$ & $\cdots$ & $1.40(20)$ & $2.5_{-0.2}^{+0.2}$ & $13_{-6}^{+13}$ \\
\hline & \multirow{2}{*}{0112800201} & 0.13 & $\cdots$ & $\cdots$ & $0.07_{-0.01}^{+0.01}$ & $1092_{-574}^{+1336}$ & $\cdots$ & $\cdots$ & $\cdots$ & $0.14_{-0.02}^{+0.03}$ & $0.8_{-0.2}^{+0.2}$ \\
\hline & & 0.20 & $0.07_{-0.01}^{+0.01}$ & $2782_{-1650}^{+4467}$ & $\cdots$ & $\cdots$ & $\cdots$ & $\cdots$ & $\cdots$ & $0.13_{-0.02}^{+0.03}$ & $1.8_{-0.4}^{+0.5}$ \\
\hline \multirow{2}{*}{29251} & \multirow{2}{*}{0205230501} & $0.09_{-0.04}^{+0.05}$ & $\begin{array}{l}0.8_{-0.1}^{+0.01} \\
\end{array}$ & $\begin{array}{c}-1000 \\
0.04_{-0.02}^{+0.04} \\
\end{array}$ & $\cdots$ & $\cdots$ & $\cdots$ & . & $0.68(29)$ & $\begin{array}{l}2.4_{-0.3}^{+0.02} \\
\end{array}$ & $3.0_{-0.4}^{+0.4}$ \\
\hline & & $0.28_{-0.08}^{+0.08}$ & $\cdots$ & $\cdots$ & $\cdots$ & $\cdots$ & $2.4_{-0.3}^{+0.3}$ & $131_{-31}^{+41}$ & $0.66(29)$ & $3.1_{-0.5}^{+0.5}$ & $6_{-1}^{+2}$ \\
\hline \multirow{2}{*}{65459} & \multirow{2}{*}{0306560301} & $0.00^{+0.06}$ & $\cdots$ & $\cdots$ & $0.11_{-0.01}^{+0.01}$ & $10_{-3}^{+4}$ & $\cdots$ & $\cdots$ & $1.19(22)$ & $0.11_{-0.01}^{+0.01}$ & $0.11_{-0.01}^{+0.01}$ \\
\hline & & $0.01_{-0.01}^{+0.07}$ & $0.14_{-0.03}^{+0.02}$ & $3_{-2}^{+17}$ & $\cdots$ & $\cdots$ & $\cdots$ & $\cdots$ & $1.06(22)$ & $0.11_{-0.02}^{+0.02}$ & $0.14_{-0.03}^{+0.13}$ \\
\hline \multirow{4}{*}{101335} & 0112830201 & $0.00+0.01$ & $\cdots$ & $\cdots$ & $0.15_{-0.01}^{+0.01}$ & $10_{-2}^{+2}$ & $\ldots$ & $\ldots$ & $1.34(52)$ & $0.44_{-0.03}^{+0.03}$ & $0.44_{-0.03}^{+0.03}$ \\
\hline & 0112000201 & $0.00^{+0.01}$ & $0.21_{-0.01}^{+0.01}$ & $1.8_{-0.4}^{+0.5}$ & 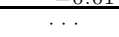 & $\cdots$ & 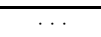 & 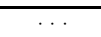 & $0.92(52)$ & $0.47_{-0.03}^{+0.03}$ & $0.47_{-0.03}^{+0.03}$ \\
\hline & 0402660201 & 0.0 & $\cdots$ & $\cdots$ & $\cdots$ & $\cdots$ & $2.1_{-0.3}^{+0.3}$ & $6_{-2}^{+2}$ & $\cdots$ & $0.3_{-0.1}^{+0.1}$ & $0.3_{-0.1}^{+0.1}$ \\
\hline & & 0.0 & $0.6_{-0.1}^{+0.2}$ & $0.010_{-0.007}^{+0.016}$ & $\cdots$ & $\cdots$ & $\cdots$ & $\cdots$ & $\cdots$ & $0.24_{-0.06}^{+0.07}$ & $0.24_{-0.06}^{+0.07}$ \\
\hline 101369 & 0112830201 & $0.00^{+0.02}$ & $\cdots$ & $\cdots$ & $0.08_{-0.01}^{+0.01}$ & $146_{-62}^{+119}$ & 2.0 & $0.8_{-0.4}^{+0.4}$ & $0.86(22)$ & $0.28_{-0.03}^{+0.03}$ & $0.28_{-0.03}^{+0.03}$ \\
\hline & & $0.00^{+0.02}$ & $0.10_{-0.01}^{+0.01}$ & $56_{-28}^{+58}$ & $\cdots$ & $\cdots$ & 2.0 & $0.8_{-0.4}^{+0.4}$ & $0.74(22)$ & $0.28_{-0.03}^{+0.03}$ & $0.28_{-0.03}^{+0.03}$ \\
\hline 105130 & 0200340101 & $0.00^{+0.01}$ & $0.60_{-0.03}^{+0.03}$ & $0.05_{-0.01}^{+0.01}$ & $\cdots$ & $\cdots$ & $\ldots$ & $\cdots$ & $0.94(138)$ & $1.21_{-0.07}^{+0.08}$ & $1.21_{-0.07}^{+0.08}$ \\
\hline & 0145800101 & $0.00_{-0.00}^{+0.01}$ & $\cdots$ & $\cdots$ & $0.12_{-0.01}^{+0.01}$ & $32_{-6}^{+7}$ & $\cdots$ & $\cdots$ & $1.19(73)$ & $0.48_{-0.03}^{+0.03}$ & $0.48_{-0.03}^{+0.03}$ \\
\hline & & $0.00_{-0.00}^{+0.03}$ & $0.16_{-0.02}^{+0.01}$ & $8_{-2}^{+9}$ & $\cdots$ & $\cdots$ & $\cdots$ & $\cdots$ & $1.10(73)$ & $0.50_{-0.04}^{+0.03}$ & $0.51_{-0.03}^{+0.15}$ \\
\hline 107102 & 0306630101 & $0.00_{-0.00}^{+0.01}$ & $\cdots$ & $\cdots$ & $0.14_{-0.01}^{+0.01}$ & $30_{-3}^{+4}$ & $\cdots$ & $\cdots$ & $1.09(142)$ & $1.05_{-0.04}^{+0.04}$ & $1.05_{-0.04}^{+0.04}$ \\
\hline & & $0.03_{-0.02}^{+0.02}$ & $0.18_{-0.01}^{+0.01}$ & $11_{-3}^{+6}$ & $\cdots$ & $\cdots$ & $\cdots$ & $\cdots$ & $1.04(142)$ & $1.06_{-0.04}^{+0.04}$ & $1.37_{-0.17}^{+0.21}$ \\
\hline & 0306630201 & $0.00_{-0.00}^{+0.02}$ & $\cdots$ & $\cdots$ & $0.13_{-0.01}^{+0.01}$ & $37_{-4}^{+4}$ & $\cdots$ & $\cdots$ & $1.05(144)$ & $0.77_{-0.03}^{+0.03}$ & $0.77_{-0.03}^{+0.03}$ \\
\hline & & $0.03_{-0.02}^{+0.02}$ & $0.15_{-0.01}^{+0.01}$ & $19_{-7}^{+11}$ & $\cdots$ & $\cdots$ & $\cdots$ & $\cdots$ & $1.04(144)$ & $0.76_{-0.03}^{+0.03}$ & $1.10_{-0.15}^{+0.20}$ \\
\hline 122979 & 0105261401 & $0.00+0.05$ & $\cdots$ & $\cdots$ & $\ldots$ & $\cdots$ & $0.9_{-0.2}^{+0.2}$ & $15_{-2}^{+2}$ & $1.07(15)$ & $2.7_{-0.5}^{+0.5}$ & $2.7_{-0.5}^{+0.5}$ \\
\hline & 0105261701 & & 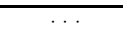 & $\cdots$ & 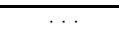 & $\cdots$ & $1.0_{-0.3}^{+0.3}$ & $10_{-3}^{+3}$ & $0.60(6)$ & $1.6_{-0.5}^{+0.0}$ & $1.6_{-0.5}^{+0.6}$ \\
\hline & 0203910101 & $0.42_{-0.06}^{+0.07}$ & $\cdots$ & $\cdots$ & $\cdots$ & $\cdots$ & $1.6_{-0.1}^{+0.1}$ & $257_{-39}^{+46}$ & $0.95(65)$ & $13_{-1}^{+1}$ & $19_{-1}^{+1}$ \\
\hline 142052 & $0402910101^{\mathrm{C}}$ & $0.41_{-0.04}^{+0.04}$ & $\cdots$ & $\cdots$ & $\cdots$ & $\cdots$ & $1.7_{-0.1}^{+0.1}$ & $123_{-11}^{+12}$ & $1.11(215)$ & $6.3_{-0.3}^{+0.3}$ & $8.8_{-0.4}^{+0.4}$ \\
\hline & 0410581901 & 0.41 & $\cdots$ & $\cdots$ & $\cdots$ & $\cdots$ & $2.3_{-0.3}^{+0.3}$ & $23_{-5}^{+5}$ & $\cdots$ & $0.5_{-0.1}^{+0.2}$ & $1.1_{-0.2}^{+0.2}$ \\
\hline & $0560181101^{\mathrm{d}}$ & $0.48_{-0.04}^{+0.05}$ & $\cdots$ & $\cdots$ & $\cdots$ & $\cdots$ & $1.9_{-0.1}^{+0.1}$ & $130_{-14}^{+15}$ & $1.00(188)$ & $5.0_{-0.3}^{+0.3}$ & $8.0_{-0.5}^{+0.5}$ \\
\hline & 0109490101 & & $\cdots$ & $\cdots$ & $\cdots$ & $\ldots$ & $1.0_{-0.3}^{+0.3}$ & $14_{-4}^{+6}$ & $0.85(14)$ & $2.0_{-0.4}^{+0.4}$ & $2.3_{-0.4}^{+0.4}$ \\
\hline & 0109490201 & & $\cdots$ & $\cdots$ & $\cdots$ & $\cdots$ & $1.3_{-0.3}^{+0.3}$ & $29_{-10}^{+14}$ & $0.87(8)$ & $2.6_{-0.6}^{+0.7}$ & $3.2_{-0.6}^{+0.7}$ \\
\hline & 0109490301 & & $\cdots$ & $\cdots$ & $\cdots$ & $\cdots$ & $0.7_{-0.2}^{+0.2}$ & $13_{-4}^{+5}$ & $0.84(20)$ & $2.9_{-0.6}^{+0.7}$ & $3.2_{-0.6}^{+0.6}$ \\
\hline 150140 & 0109490401 & $0.6_{-0.2}^{+}$ & $\cdots$ & $\cdots$ & $\cdots$ & $\cdots$ & $0.9_{-0.2}^{+0.3}$ & $15_{-4}^{+6}$ & $0.69(16)$ & $2.3_{-0.5}^{+0.5}$ & $2.6_{-0.5}^{+0.5}$ \\
\hline & 0109490501 & & $\cdots$ & $\cdots$ & $\cdots$ & $\cdots$ & $1.0_{-0.3}^{+0.3}$ & $13_{-4}^{+6}$ & $1.04(13)$ & $1.8_{-0.4}^{+0.5}$ & $2.1_{-0.5}^{+0.5}$ \\
\hline & 0109490601 & & $\cdots$ & $\cdots$ & $\cdots$ & $\cdots$ & $0.8_{-0.2}^{+0.2}$ & $\begin{array}{l}12_{-3}^{+5} \\
\end{array}$ & $0.65(17)$ & $2.1_{-0.4}^{+0.5}$ & $2.4_{-0.4}^{+0.5}$ \\
\hline & $6291(\mathrm{CXO})$ & $0.8_{-0.3}^{+0.3}$ & $\cdots$ & $\cdots$ & $\cdots$ & $\cdots$ & $1.2_{-0.3}^{+0.3}$ & $30_{-11}^{+18}$ & $0.53(18)$ & $3.1_{-0.5}^{+0.5}$ & $3.8_{-0.4}^{+0.4}$ \\
\hline 165335 & 0149610401 & $0.60_{-0.06}^{+0.07}$ & $\cdots$ & $\cdots$ & $\cdots$ & $\cdots$ & $2.1_{-0.1}^{+0.1}$ & $\begin{array}{l}251_{-33}^{+39} \\
\end{array}$ & $1.04(108)$ & $6.8_{-0.5}^{+0.5}$ & $13_{-1}^{+2}$ \\
\hline 174295 & 0200450301 & $1.3_{-0.2}^{+0.3}$ & $\cdots$ & $\cdots$ & $\cdots$ & $\cdots$ & $1.9_{-0.2}^{+0.2}$ & $482_{-131}^{+194}$ & $1.16(50)$ & $15_{-2}^{+2}$ & $29_{-4}^{+7}$ \\
\hline 183876 & 0311190101 & $0.00+0.06$ & $\cdots$ & $\cdots$ & $\begin{array}{c}0.17_{-0.02}^{+0.01} \\
\end{array}$ & $3_{-1}^{+5}$ & $\begin{array}{r}1.3_{-0.4}^{+1.0} \\
\end{array}$ & $2_{-1}^{+2}$ & $1.19(46)$ & $0.43_{-0.07}^{+0.08}$ & $0.44_{-0.07}^{+0.13}$ \\
\hline & & $0.06_{-0.04}^{+0.06}$ & $0.21_{-0.03}^{+0.04}$ & $2_{-1}^{+4}$ & $\cdots$ & $\cdots$ & $1.3_{-0.8}^{+0.9}$ & $2_{-1}^{+2}$ & $1.21(46)$ & $0.43_{-0.07}^{+0.08}$ & $0.61_{-0.14}^{+0.24}$ \\
\hline 187831 & 0204610101 & $0.00^{+0.03}$ & $\cdots$ & $\cdots$ & $0.12_{-0.01}^{+0.01}$ & $21_{-4}^{+20}$ & $\cdots$ & $\cdots$ & $1.24(68)$ & $0.31_{-0.02}^{+0.02}$ & $0.31_{-0.02}^{+0.10}$ \\
\hline & & $0.03_{-0.03}^{+0.03}$ & $0.14_{-0.01}^{+0.01}$ & $12_{-6}^{+15}$ & $\cdots$ & $\cdots$ & $\cdots$ & $\cdots$ & $1.13(68)$ & $0.31_{-0.02}^{+0.02}$ & $0.44_{-0.10}^{+0.16}$ \\
\hline & 0505720201 & $0.15_{-0.06}^{+0.06}$ & $\cdots$ & $\cdots$ & $\cdots$ & $\cdots$ & $2.3_{-0.3}^{+0.3}$ & $85_{-17}^{+22}$ & $1.15(26)$ & $2.6_{-0.4}^{+0.4}$ & $4.2_{-0.7}^{+1.0}$ \\
\hline & 0505720301 & $0.00^{+0.05}$ & $\cdots$ & $\cdots$ & $\cdots$ & $\cdots$ & $1.9_{-0.2}^{+0.3}$ & $30_{-4}^{+8}$ & $0.96(14)$ & $1.8_{-0.4}^{+0.3}$ & $1.7_{-0.3}^{+0.2}$ \\
\hline & 0505720401 & $0.00+0.09$ & $\cdots$ & $\cdots$ & $\cdots$ & $\cdots$ & $1.3_{-0.2}^{+0.2}$ & $25_{-4}^{+4}$ & $1.32(8)$ & $2.5_{-0.6}^{+0.7}$ & $2.5_{-0.6}^{+0.7}$ \\
\hline & 0505720501 & $0.04_{-0.04}^{+0.07}$ & $\cdots$ & $\cdots$ & $\cdots$ & $\cdots$ & $1.6_{-0.3}^{+0.5}$ & $113_{-24}^{+32}$ & $0.85(14)$ & $8_{-1}^{+1}$ & $8_{-1}^{+1}$ \\
\hline & 0505720601 & $0.13_{-0.05}^{+0.06}$ & $\cdots$ & $\cdots$ & $\cdots$ & $\cdots$ & $1.8_{-0.2}^{+0.2}$ & $127_{-23}^{+28}$ & $0.76(37)$ & $6.5_{-0.8}^{+0.9}$ & $8.1_{-0.8}^{+0.8}$ \\
\hline & 0551690201 & $0.08_{-0.05}^{+0.06}$ & 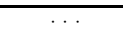 & 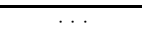 & 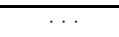 & $\cdots$ & $1.7_{-0.2}^{+0.3}$ & $73_{-15}^{+19}$ & $1.06(24)$ & $4.3_{-0.7}^{+0.8}$ & $5.0_{-0.6}^{+0.6}$ \\
\hline & 0551690301 & $0.09_{-0.06}^{+0.08}$ & $\cdots$ & $\cdots$ & $\cdots$ & $\cdots$ & $1.9_{-0.3}^{+0.4}$ & $52_{-13}^{+18}$ & $0.74(15)$ & $2.5_{-0.5}^{+0.6}$ & $3.0_{-0.5}^{+0.5}$ \\
\hline & 0551690401 & $0.1_{-0.1}^{+0.1}$ & $\cdots$ & $\cdots$ & $\cdots$ & $\cdots$ & $1.7_{-0.5}^{+0.5}$ & $87_{-30}^{+48}$ & $\cdots$ & $5_{-1}^{+2}$ & $6_{-1}^{+2}$ \\
\hline & 0551690501 & $0.03_{-0.03}^{+0.12}$ & $\cdots$ & $\cdots$ & $\cdots$ & $\cdots$ & $2.1_{-0.4}^{+0.7}$ & $47_{-11}^{+24}$ & $1.09(6)$ & $2.2_{-0.6}^{+0.6}$ & $2.5_{-0.5}^{+1.0}$ \\
\hline & 0551690601 & $0.2_{-0.2}^{+0.2}$ & $\cdots$ & $\cdots$ & $\cdots$ & $\cdots$ & $2.8_{-0.8}^{+1.1}$ & $57_{-25}^{+55}$ & $\cdots$ & $1.2_{-0.4}^{+0.6}$ & $3_{-1}^{+6}$ \\
\hline 222148 & 0600660201 & $0.10_{-0.05}^{+0.06}$ & $\cdots$ & $\cdots$ & $\cdots$ & $\cdots$ & $1.8_{-0.2}^{+0.2}$ & $96_{-17}^{+21}$ & $1.06(27)$ & $5.4_{-0.8}^{+0.9}$ & $6.4_{-0.7}^{+0.8}$ \\
\hline & 0600660301 & $0.14_{-0.06}^{+0.07}$ & $\cdots$ & $\cdots$ & $\cdots$ & $\cdots$ & $2.0_{-0.3}^{+0.3}$ & $106_{-22}^{+28}$ & $1.03(21)$ & $4.3_{-0.7}^{+0.7}$ & $5.9_{-0.8}^{+0.9}$ \\
\hline & 0600660401 & $0.10_{-0.06}^{+0.07}$ & $\cdots$ & $\cdots$ & $\cdots$ & 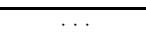 & $1.8_{-0.2}^{+0.3}$ & $\begin{array}{l}94_{-19}^{+24} \\
\end{array}$ & $0.42(21)$ & $\begin{array}{l}\frac{-0.1}{+0.8} \\
4.8-8\end{array}$ & $5.8_{-0.7}^{+0.8}$ \\
\hline & 0600660501 & $0.08_{-0.06}^{+0.07}$ & $\cdots$ & $\cdots$ & $\cdots$ & $\cdots$ & $1.8_{-0.3}^{+0.3}$ & $90_{-21}^{+28}$ & $0.97(13)$ & $4.8_{-0.8}^{+0.9}$ & $5.7_{-0.7}^{+0.8}$ \\
\hline & 0600660601 & $0.14_{-0.08}^{+0.09}$ & $\cdots$ & $\cdots$ & $\cdots$ & $\cdots$ & $2.0_{-0.3}^{+0.4}$ & $105_{-27}^{+38}$ & $0.90(14)$ & $4.2_{-0.8}^{+0.9}$ & $5.8_{-0.9}^{+1.3}$ \\
\hline & 0650560201 & $0.11_{-0.04}^{+0.05}$ & $\cdots$ & $\cdots$ & $\cdots$ & $\cdots$ & $1.7_{-0.2}^{+0.2}$ & $112_{-17}^{+20}$ & $1.23(43)$ & $6.3_{-0.8}^{+0.8}$ & $7.6_{-0.7}^{+0.7}$ \\
\hline & 0650560301 & $0.10_{-0.04}^{+0.04}$ & $\cdots$ & $\cdots$ & $\cdots$ & $\cdots$ & $1.8_{-0.1}^{+0.2}$ & $113_{-15}^{+18}$ & $0.72(51)$ & $5.9_{-0.6}^{+0.6}$ & $7.2_{-0.6}^{+0.6}$ \\
\hline & 0650560401 & $0.15_{-0.06}^{+0.07}$ & $\cdots$ & $\cdots$ & $\cdots$ & $\cdots$ & $2.0_{-0.2}^{+0.3}$ & $121_{-23}^{+29}$ & $0.88(27)$ & $4.7_{-0.7}^{+0.8}$ & $6.6_{-0.8}^{+1.0}$ \\
\hline & 0650560501 & $0.14_{-0.06}^{+0.07}$ & $\cdots$ & $\cdots$ & $\cdots$ & $\cdots$ & $1.9_{-0.2}^{+0.3}$ & $136_{-27}^{+35}$ & $1.02(21)$ & $6.3_{-0.9}^{+1.0}$ & $8.2_{-0.8}^{+0.9}$ \\
\hline & 0650560601 & $0.08_{-0.04}^{+0.04}$ & $\cdots$ & $\cdots$ & $\cdots$ & $\cdots$ & $1.8_{-0.2}^{+0.2}$ & $114_{-16}^{+19}$ & $0.70(41)$ & $6.3_{-0.7}^{+0.7}$ & $7.4_{-0.7}^{+0.7}$ \\
\hline & 0672130101 & $0.13_{-0.03}^{+0.03}$ & $\cdots$ & $\cdots$ & $\cdots$ & $\cdots$ & $1.8_{-0.1}^{+0.1}$ & $117_{-12}^{+14}$ & $0.85(74)$ & $6.2_{-0.4}^{+0.5}$ & $7.7_{-0.4}^{+0.4}$ \\
\hline & 0012100101 & $0.05_{-0.03}^{+0.04}$ & $0.6_{-0.1}^{+0.2}$ & $0.10_{-0.07}^{+0.18}$ & $1.4_{-0.3}^{+1.0}$ & $0.008_{-0.007}^{+0.012}$ & $\cdots$ & $\cdots$ & $0.85(71)$ & $5.8_{-0.5}^{+0.8}$ & $6.3_{-0.6}^{+0.7}$ \\
\hline & 0672130601 & $0.13_{-0.03}^{+0.04}$ & $\cdots$ & $\cdots$ & $\cdots$ & $\cdots$ & $1.8_{-0.1}^{+0.1}$ & $118_{-13}^{+15}$ & $0.71(70)$ & $6.2_{-0.5}^{+0.5}$ & $7.7_{-0.4}^{+0.4}$ \\
\hline & 0672130701 & $0.11_{-0.03}^{+0.03}$ & $\cdots$ & $\cdots$ & $\cdots$ & $\cdots$ & $1.7_{-0.1}^{+0.1}$ & $111_{-12}^{+14}$ & $1.08(70)$ & $6.4_{-0.5}^{+0.5}$ & $7.7_{-0.4}^{+0.4}$ \\
\hline
\end{tabular}


TABLE 3 - Continued

\begin{tabular}{|c|c|c|c|c|c|c|c|c|c|c|c|}
\hline $\begin{array}{r}\text { SRCID } \\
(1)\end{array}$ & $\begin{array}{c}\text { Obs ID } \\
(2)\end{array}$ & $\begin{array}{c}\left(10^{22}{ }_{\mathrm{cm}}^{N_{\mathrm{H}}}-2\right) \\
(3)\end{array}$ & $\begin{array}{c}k T_{\mathrm{MCD}} \\
(\mathrm{keV}) \\
(4)\end{array}$ & $\begin{array}{c}N_{\mathrm{MCD}} \\
(5)\end{array}$ & $\begin{array}{c}k T_{\mathrm{BB}} \\
(\mathrm{keV}) \\
(6)\end{array}$ & $\begin{array}{c}N_{\mathrm{BB}} \\
(7)\end{array}$ & $\begin{array}{c}\Gamma_{\mathrm{PL}} \\
(8)\end{array}$ & $\begin{array}{c}N_{\mathrm{PL}} \\
\left(10^{-6}\right) \\
(9)\end{array}$ & $\begin{array}{c}\chi_{\nu}^{2}(\nu)^{\mathrm{a}} \\
(10)\end{array}$ & $\begin{array}{r}F_{\text {abs }} \\
\left(10^{-13}\right. \\
(11)\end{array}$ & $\mathrm{erg} \mathrm{s}^{-1} \underset{\left.\mathrm{cm}^{-2}\right)}{(12)}$ \\
\hline & 0672130501 & $0.10_{-0.05}^{+0.06}$ & $\cdots$ & $\cdots$ & $\cdots$ & $\cdots$ & $1.6_{-0.2}^{+0.2}$ & $88_{-17}^{+22}$ & $1.11(25)$ & $5.6_{-0.8}^{+0.8}$ & $6.5_{-0.6}^{+0.6}$ \\
\hline 222243 & 0405320801 & $0.01_{-0.01}^{+0.06}$ & $0.4_{-0.1}^{+0.1}$ & $0.3_{-0.2}^{+1.1}$ & $1.5_{-0.1}^{+0.1}$ & $0.019_{-0.004}^{+0.005}$ & $\cdots$ & $\cdots$ & $0.94(118)$ & $9.4_{-0.7}^{+0.7}$ & $9.5_{-0.7}^{+0.7}$ \\
\hline 241580 & 0556300101 & $0.2_{-0.1}^{+0.1}$ & $\cdots$ & $\cdots$ & $\cdots$ & $\cdots$ & $1.6_{-0.2}^{+0.2}$ & $18_{-4}^{+6}$ & $1.07(26)$ & $1.1_{-0.2}^{+0.2}$ & $1.3_{-0.2}^{+0.2}$ \\
\hline
\end{tabular}

Note. - Columns: (1) 2XMM-DR3 Unique source index; (2) observation ID; (3) absorption column density; (4) the MCD temperature; (5) the MCD normization $N_{\mathrm{MCD}} \equiv\left(\left(R_{\mathrm{MCD}} / \mathrm{km}\right) /(D / 10 \mathrm{kpc})\right)^{2} \cos \theta$, where $R_{\mathrm{MCD}}$ is the apparent inner disk radius, $D$ is the source distance, $\theta$ is the disk inclination; (6) the BB temperature; (7) the BB normization $N_{\mathrm{BB}} \equiv\left(\left(R_{\mathrm{BB}} / \mathrm{km}\right) /(D / 10 \mathrm{kpc})\right)^{2}$, where $R_{\mathrm{BB}}$ is the source radius; (8) the PL photon index; (9) the PL normalization; (10)-(11) the $0.3-10$
keV absorbed and unabsorbed flux, respectively. All the fits used spectra within $0.3-10 \mathrm{keV}$. The $N_{\mathrm{H}}$ values shared by multiple observations was obtained from our $\mathrm{keV}$ absorbed and unabsorbed flux, respectively. All the fits used spectra within $0.3-10 \mathrm{keV}$. The $N_{\mathrm{H}}$ values shared by
simultaneous fits of these observations with this parameter tied together. Parameters without errors were fixed in the fits.

a The $\chi^{2}$ values are listed only for fits using $\chi^{2}$ statistic.

$\mathrm{b}$ These fits included a narrow Gaussian line, with $\sigma_{\mathrm{Ga}}$ fixed at $0.01 \mathrm{keV}$ and the best-fitting centroid energy $E_{\mathrm{Ga}}=0.87 \pm 0.02 \mathrm{keV}$.

C This fit included a narrow Gaussian line, with the best-fitting $E_{\mathrm{Ga}}=6.7 \pm 0.1 \mathrm{keV}, \sigma_{\mathrm{Ga}}=0.2 \pm 0.1 \mathrm{keV}$ and $N_{\mathrm{Ga}}=(4 \pm 1) \times 10-6$. The EW is $1.0 \pm 0.3 \mathrm{keV}$.

$\mathrm{d}$ This fit included a narrow Gaussian line, with the best-fitting $E_{\mathrm{Ga}}=6.6 \pm 0.1 \mathrm{keV}, \sigma_{\mathrm{Ga}}=0.2 \pm 0.2 \mathrm{keV}$ and $N_{\mathrm{Ga}}=(3 \pm 1) \times 10^{-6}$. The EW is $0.9 \pm 0.4 \mathrm{keV}$. There is also some residual at energy around $8 \mathrm{keV}$, which is mostly likely due to the pn instrument background line (Cu-K $\alpha$ ). We thus excluded the pn data between $7.8-8.2$ $\mathrm{keV}$ for this spectral fit.

TABLE 4

THE CANDIDATE SDSS COUNTERPARTS

\begin{tabular}{ccccccccc}
\hline \hline SRCID & SDSS & Dxo cl & $u$ & $g$ & $r$ & $i$ & $z$ \\
$(1)$ & $(2)$ & $(3)$ & $(4)$ & $(5)$ & $(6)$ & $(7)$ & $(8)$ & $(9)$ \\
\hline 101335 & J121028.95+391748.9 & 0.2 & 6 & $22.64 \pm 0.40$ & $22.22 \pm 0.12$ & $21.67 \pm 0.10$ & $21.68 \pm 0.14$ & $21.07 \pm 0.26$ \\
101369 & $\mathrm{~J} 121034.99+393122.9$ & 0.6 & 3 & $22.00 \pm 0.16$ & $20.49 \pm 0.02$ & $19.49 \pm 0.01$ & $19.10 \pm 0.01$ & $18.79 \pm 0.04$ \\
107102 & $\mathrm{~J} 123103.24+110648.6$ & 0.5 & 3 & $22.21 \pm 0.30$ & $20.75 \pm 0.04$ & $20.12 \pm 0.03$ & $19.71 \pm 0.03$ & $19.45 \pm 0.08$ \\
174295 & $\mathrm{~J} 203353.67+410717.2$ & 0.1 & 6 & $25.91 \pm 0.48$ & $24.47 \pm 0.47$ & $21.44 \pm 0.07$ & $19.65 \pm 0.02$ & $18.28 \pm 0.03$ \\
\hline
\end{tabular}

Note. - Columns are as follows. (1): 2XMM-DR3 Unique source index; (2) SDSS8 source designation; (3) X-ray-optical separation (arcsec) (4) SDSS object class ("3": galaxy; "6": star); (5)-(9): SDSS magnitudes. 